NOTICE: this is the author's version of a work that was accepted for publication in Computers \& Geosciences. Changes resulting from the publishing process, such as peer review, editing, corrections, structural formatting, and other quality control mechanisms may not be reflected in this document. Changes may have been made to this work since it was submitted for publication. A definitive version was subsequently published in Computers \& Geosciences, Vol. 54 (2013). DOI: 10.1016/j.cageo.2013.01.014 


\title{
Ps-LAMBDA: \\ Ambiguity Success Rate Evaluation Software for Interferometric Applications
}

\author{
Sandra Verhagen ${ }^{\mathrm{a}, *}$, Bofeng Li $^{\mathrm{b}}$, Peter J.G. Teunissen ${ }^{\mathrm{b}, \mathrm{a}}$ \\ ${ }^{a}$ Mathematical Geodesy and Positioning, Delft University of Technology \\ ${ }^{b}$ GNSS Research Centre, Department of Spatial Sciences, Curtin University
}

\begin{abstract}
Integer ambiguity resolution is the process of estimating the unknown ambiguities of carrier-phase observables as integers. It applies to a wide range of interferometric applications of which Global Navigation Satellite System (GNSS) precise positioning is a prominent example. GNSS precise positioning can be accomplished anytime and anywhere on Earth, provided that the integer ambiguities of the very precise carrier-phase observables are successfully resolved. As wrongly resolved ambiguities may result in unacceptably large position errors, it is crucial that one is able to evaluate the probability of correct integer ambiguity estimation. This ambiguity success rate depends on the underlying mathematical model as well as on the integer estimation method used. In this contribution, we present the Matlab toolbox Ps-LAMBDA for the evaluation of the ambiguity success rates. It allows users to evaluate all available success rate bounds and approximations for different integer estimators. An assessment of the sharpness of the bounds and approximations is given as well. Furthermore, it is shown how the toolbox
\end{abstract}

*Tel: +31 15 2784545/Fax: +31 15 2783711

Email address: A. A.Verhagen@TUDelft.nl (Sandra Verhagen) 
can be used to assess the integer ambiguity resolution performance for design and research purposes, so as to study for instance the impact of using different GNSS systems and/or different measurement scenarios.

Keywords: Ambiguity Success Rate, GNSS, Interferometry, Integer Estimation, Ps-LAMBDA

\section{Introduction}

The range of applications depending on precise positioning in real-time with a Global Navigation Satellite System (GNSS) has been growing dramatically in 4 the past decades, and will continue to do so with the advent of more signals and systems by means of modernized GPS, the European Galileo and the Chinese 6 Compass. The applications range from navigation and geodetic surveying to Earth observation, construction, and safety-of-life navigation.

$8 \quad$ All these applications have in common that they rely on the very precise GNSS carrier-phase observations for precise (and real-time) positioning. These observations are ambiguous by an unknown integer number of cycles. Only if the ambiguities can be resolved correctly, is it possible to obtain accuracies at centimeterlevel or below. It is therefore important to assess the probability of correct integer estimation, called the success rate. Although a variety of success rate bounds have been developed, no standard software exists to evaluate these bounds for different integer estimation methods. In this contribution, we introduce the new Matlab tool Ps-LAMBDA for the evaluation of interferometric ambiguity success rates.

Here we briefly address some specific examples of the broad-ranging geoscience applications for which correct integer ambiguity estimation is crucial. For these applications, the success rate tool is valuable, not only for deciding 
on whether or not to fix the ambiguities in (real-time) data processing algorithms, but also for design and research purposes, so as to study for instance, the impact or potential of using different GNSS systems, different measurement scenarios and/or choice of signals, receivers or baseline lengths.

As a first example, we mention the GNSS Real-Time Kinematic (RTK) technique, (Odijk, 2002; Li and Teunissen, 2011; Euler et al., 2004; Takac and Zelzer, 2008). It is widely used for mapping, geodetic surveying and network applications, (Blewitt, 1989; Bock, 1996; Strang and Borre, 1997; Leick, 2004; HofmannWellenhof et al., 2008; Teunissen and Kleusberg, 1998). With GNSS-RTK, centimeterlevel positioning in real-time can be achieved based on relative positioning to one or more base stations. Cancellation or mitigation of common error sources allows for improved precision, but centimeter-level accuracies can only be achieved after the successful estimation of the integer ambiguities.

Recently, the RTK-technique has been extended to the concept of Precise Point Positioning (PPP), (Heroux and Kouba, 1995; Zumberge et al., 1997). With PPP, satellite orbit and clock corrections from a global network of receivers are used to enable subdecimeter accuracies. This has the advantage that PPP does not rely on a dense network of receivers, and thus can be used anywhere on Earth. The newly developed PPP-RTK technique allows to exploit the precise GNSS carrier-phase measurements, such that higher precisions and shorter convergence times become feasible, (Odijk et al., 2012; Geng et al., 2012; Collins et al., 2009; Laurichesse et al., 2009). Hence, also for PPR-RTK, reliable integer estimation is crucial.

Another example is GNSS-based georeferencing as used in many remote sensing applications. The remote sensing platforms range from survey ships, to airborne and Unmanned Airborne Vehicles (Everaerts, 2008; Rieke et al., 2011), 
and even spaceborne platforms, (Buist et al., 2010; Kroes et al., 2005; Leung and Montenbruck, 2005; Huber et al., 2010; Nadarajah et al., 2012). GNSS allows for precise positioning and attitude determination of the platforms, in real-time or in post-processing mode. In either case, to enable precise georeferencing, successful carrier-phase ambiguity resolution is needed.

Integer ambiguity resolution is also important in the quality control of GNSS data. Loss-of-lock and/or high receiver dynamics may cause integer cycle slips in the carrier phase data. Successful cycle slip detection and repair is therefore important to maintain the integrity of the data (deLacy et al., 2011; Dai et al., 2009; Wu et al., 2010).

Also several non-positioning applications of GNSS can be given as examples. For instance, the use of GNSS stations at fixed locations for deformation monitoring and change detection, e.g. in tectonic active regions, near volcanoes and in areas susceptible to deformation, (Dong and Bock, 1989; Fernandes et al., 2004; Michel et al., 2001). Or the use of observed GNSS path delays for atmospheric profiling, integrated water vapor measurement and creating ionosphere maps, (Bevis et al., 1992; Van der Hoeven et al., 2002; Schon and Brunner, 2008; Todorova et al., 2008; Wickert et al., 2009). In all these applications, the precise carrierphase observables are needed, but the signal of interest can only be extracted once the unknown integer ambiguities are resolved.

Next to GNSS, Ps-LAMBDA also applies to all other interferometric techniques for which the integer ambiguity resolution problem plays a role. Interferometric Synthetic Aperture Radar (InSAR) is one example. The associated interferometric phase observations can be described by a linear function of topographic height, surface deformation, and the integer ambiguity parameters. Precise esti- 
mation of the surface deformation is thus dependent on successful resolution of the integer parameters, (Hanssen et al., 2001). Similarly, the fringe phase observations from Very Long Baseline Interferometry (VLBI) include unknown integer ambiguities, which need to be resolved in order to achieve the $1 \mathrm{~mm}$ global positioning accuracy, (Hobiger et al., 2009). As another example, we mention the use of acoustic waves for precise positioning, e.g. for indoor and underwater applications, (Das Neves Viegas and Cunha, 2007). Again integer ambiguity resolution is the key.

As the above examples show, the evaluation of the integer ambiguity success rate is important for a wide range of interferometric applications. In this contribution, we use GNSS and its models to present and describe the Matlab toolbox Ps-LAMBDA. Section 2 presents the basic GNSS model and the essence of correct integer ambiguity estimation. Section 3 reviews the three integer estimators integer rounding (IR), integer bootstrapping (IB) and integer least squares (ILS). In Section 4, the multivariate success rate is defined and the Ps-LAMBDA software to evaluate the success rate is described. In Section 5 the available success rate bounds and approximations of IR, IB and ILS are presented, together with an assessment of their performance. Section 6 presents an example of how the toolbox can be used to assess the performance potential of the American GPS, the European Galileo, and the Chinese Compass satellite systems. 
Table 1: Overview of GNSS frequencies of open signals.

\begin{tabular}{l|l|ccc}
\hline Band & Frequency & GPS & Galileo & Compass \\
\hline L1 & $1575.42 \mathrm{MHz}$ & $\mathrm{X}$ & $\mathrm{X}$ & \\
B1 & $1561.098 \mathrm{MHz}$ & & & $\mathrm{X}$ \\
L2 & $1227.60 \mathrm{MHz}$ & $\mathrm{X}$ & & \\
E5b/B2 & $1207.14 \mathrm{MHz}$ & & $\mathrm{X}$ & $\mathrm{X}$ \\
L5/E5a & $1176.45 \mathrm{MHz}$ & $\mathrm{X}$ & $\mathrm{X}$ & \\
\hline
\end{tabular}

\section{GNSS model and integer estimation}

\subsection{GNSS observation equations}

Precise GNSS positioning relies on the carrier-phase observations, which can be observed with millimeter precision versus decimeter precision for the pseudorange observations. The frequencies of the GPS, Galileo and Compass open signals are given in Table 1. Glonass is not considered because ambiguity resolution is generally not applied as Glonass applies Frequency Division Multiple Access.

The pseudorange and carrier-phase observables on frequency $j$ and satellitereceiver pair $s-r$ at epoch $t$ are denoted as $p_{r, j}^{s}(t)$ and $\phi_{r, j}^{s}(t)$, respectively. Their observation equations are formulated as , cf.(Hofmann-Wellenhof et al., 2001; Teunissen and Kleusberg, 1998; Leick, 2004; Strang and Borre, 1997; Misra and Enge, 2001):

$$
p_{r, j}^{s}(t)=\rho_{r}^{s}(t)+T_{r}^{s}(t)+\mu_{j} I_{r}^{s}(t)+c d t_{r, j}^{s}(t)+e_{r}^{s}(t)
$$

$$
\phi_{r, j}^{s}(t)=\rho_{r}^{s}(t)+T_{r}^{s}(t)-\mu_{j} I_{r}^{s}(t)+c \delta t_{r, j}^{s}(t)+\lambda_{j} M_{r, j}^{s}+\epsilon_{r}^{s}(t)
$$

where $\rho_{r}^{s}$ is the satellite-receiver range, $T_{r}^{s}$ is the troposphere delay, $I_{r}^{s}$ is the ionosphere delay, $d t_{r, j}^{s}$ and $\delta t_{r, j}^{s}$ are the pseudo-range and carrier-phase satellite-receiver 
clock biases, $M_{r, j}^{s}$ is the time-invariant carrier-phase ambiguity, $c$ is the speed of light, $\lambda_{j}$ is the wavelength for frequency $j, \mu_{j}=\left(\lambda_{j} / \lambda_{1}\right)^{2}$, and $e_{r}^{s}$ and $\epsilon_{r}^{s}$ are the remaining error terms, respectively. The real-valued carrier-phase ambiguity $M_{r, j}^{s}=\phi_{r, j}\left(t_{0}\right)+\phi_{j}^{s}\left(t_{0}\right)+N_{r, j}^{s}$ is the sum of the initial satellite-receiver phases and the integer ambiguity $N_{r, j}^{s}$.

The structure of the observation equations of the pseudorange and carrierphase observables is the same, only that the latter contains an ambiguity term. This implies that if the ambiguities can be resolved, the carrier-phase observations will start to act as very precise pseudorange observations.

The initial phases and clock biases present in Eq.(1) can be eliminated through differencing the observation equations. The so-called double differenced (DD) observation equations, using simultaneous observations from two receivers and two satellites, take the form:

$$
\begin{aligned}
& p_{q r, j}^{l s}(t)=\rho_{q r}^{l s}(t)+T_{q r}^{l s}(t)+\mu_{j} I_{q r}^{l s}(t)+e_{q r}^{l s}(t) \\
& \phi_{q r, j}^{l s}(t)=\rho_{q r}^{l s}(t)+T_{q r}^{l s}(t)-\mu_{j} I_{q r}^{l s}(t)+\lambda_{j} N_{q r, j}^{l s}+\epsilon_{q r}^{l s}(t)
\end{aligned}
$$

where $p_{q r, j}^{l s}(t)=\left(p_{r, j}^{s}(t)-p_{r, j}^{l}(t)\right)-\left(p_{q, j}^{s}(t)-p_{q, j}^{l}(t)\right)$, and similar notation for the other DD variates. The DD troposphere slant delays are usually reduced to a single DD zenith delay $T_{q r}^{\text {zenith }}$ by means of mapping functions. The DD ionosphere delays can usually be neglected for baselines shorter than $15 \mathrm{~km}$. For longer baselines, a priori ionosphere corrections can be used. In that case the uncertainty of those corrections should be taken into account.

Under the assumption that the error terms $e_{q r}^{l s}$ and $\epsilon_{q r}^{l s}$ in Eq.(2) are zero-mean variables, the observation equations can be used to set up a mixed integer linear model, as some of the parameters are reals and others are integer.

The observation equations in Eq.(2) are parameterized in terms of the satellite- 
receiver ranges $\rho_{q r}^{l s}(t)$, which depend on both the satellite and receiver positions. Assuming the satellite orbits known, these ranges can be linearized with respect to the unknown receiver coordinates. The linearized observation equations obtained in this way are then parameterized in terms of the between-receiver baseline vector increments, and the model is an example of a mixed integer linearized model.

\subsection{Solving the GNSS model}

The mixed integer linear(ized) model can now be defined as:

$$
\boldsymbol{y} \sim \boldsymbol{N}\left(\boldsymbol{A} \boldsymbol{a}+\boldsymbol{B} \boldsymbol{b}, Q_{y y}\right), \boldsymbol{a} \in \mathbb{Z}^{n}, \boldsymbol{b} \in \mathbb{R}^{p}
$$

The notation " $\sim$ " is used to describe "distributed as". The $m$-vector $\boldsymbol{y}$ contains the pseudorange and carrier-phase observables, the $n$-vector $\boldsymbol{a}$ contains the DD integer ambiguities, $\boldsymbol{b}$ is the real-valued parameter vector of length $p$, including baseline or position components and possibly tropospheric and ionospheric delay parameters. The coefficient matrices are $\boldsymbol{A} \in \mathbb{R}^{m \times n}$ and $\boldsymbol{B} \in \mathbb{R}^{m \times p}$, with $[\boldsymbol{A} \boldsymbol{B}$ ] of full column rank. The variance-covariance (VC-) matrix $\boldsymbol{Q}_{\boldsymbol{y} y}$ is an $m \times m$ positive definite matrix. In most GNSS applications, the underlying distribution is assumed to be the multivariate normal distribution.

In general, a three-step procedure is employed to solve model (3) based on the least squares criterion. In practice, a user may want to include a validation step after step 1 and step 2 .

\section{Step 1: Float solution}

In the first step, the integer property of the ambiguities $\boldsymbol{a}$ is discarded and the so-called float solution can be obtained with standard least squares:

$$
\left[\begin{array}{c}
\hat{a} \\
\hat{b}
\end{array}\right]=\left(\left[\begin{array}{c}
A^{T} \\
B^{T}
\end{array}\right] Q_{y y}^{-\mathbf{1}}\left[\begin{array}{ll}
A & B
\end{array}\right]\right)^{-1}\left[\begin{array}{c}
A^{T} \\
B^{T}
\end{array}\right] \boldsymbol{Q}_{y y}^{-\mathbf{1}} \boldsymbol{y}
$$


171 This solution is referred to as the fixed baseline solution.

$$
\left[\begin{array}{c}
\hat{a} \\
\hat{b}
\end{array}\right] \sim N\left(\left[\begin{array}{l}
\boldsymbol{a} \\
\boldsymbol{b}
\end{array}\right],\left[\begin{array}{ll}
\boldsymbol{Q}_{\hat{a} \hat{a}} & \boldsymbol{Q}_{\hat{a} \hat{b}} \\
\boldsymbol{Q}_{\hat{b} \hat{a}} & \boldsymbol{Q}_{\hat{b} \hat{b}}
\end{array}\right]\right)
$$
respectively.

\section{Step 2: Integer estimation} responding integer ambiguity estimate, denoted as

$$
\check{\boldsymbol{a}}=\mathcal{I}(\hat{\boldsymbol{a}})
$$
detail in the following subsections.

\section{Step 3: Fixed solution} solved in the first step are updated using the fixed integer parameters,

$$
\check{b}=\hat{b}-Q_{\hat{b} \hat{a}} Q_{\hat{a} \hat{a}}^{-1}(\hat{a}-\check{a})
$$

$\boldsymbol{Q}_{\hat{a} \hat{a}}$ and $\boldsymbol{Q}_{\hat{b} \hat{b}}$ are the VC-matrices of the float ambiguity and baseline estimators,

In the second step, the float ambiguity estimate $\hat{\boldsymbol{a}}$ is used to compute the cor-

with $\mathcal{I}: \mathbb{R}^{n} \longmapsto \mathbb{Z}^{n}$ the integer mapping from the $n$-dimensional space of reals to the $n$-dimensional space of integers. In this step, there are different choices of mapping function $\mathcal{I}$ possible, which correspond to the different integer estimation methods. Popular choices are integer least squares (ILS), integer bootstrapping (IB) and integer rounding (IR). Each of the methods will be discussed in more

In the third step, the float solution of the remaining real-valued parameters 


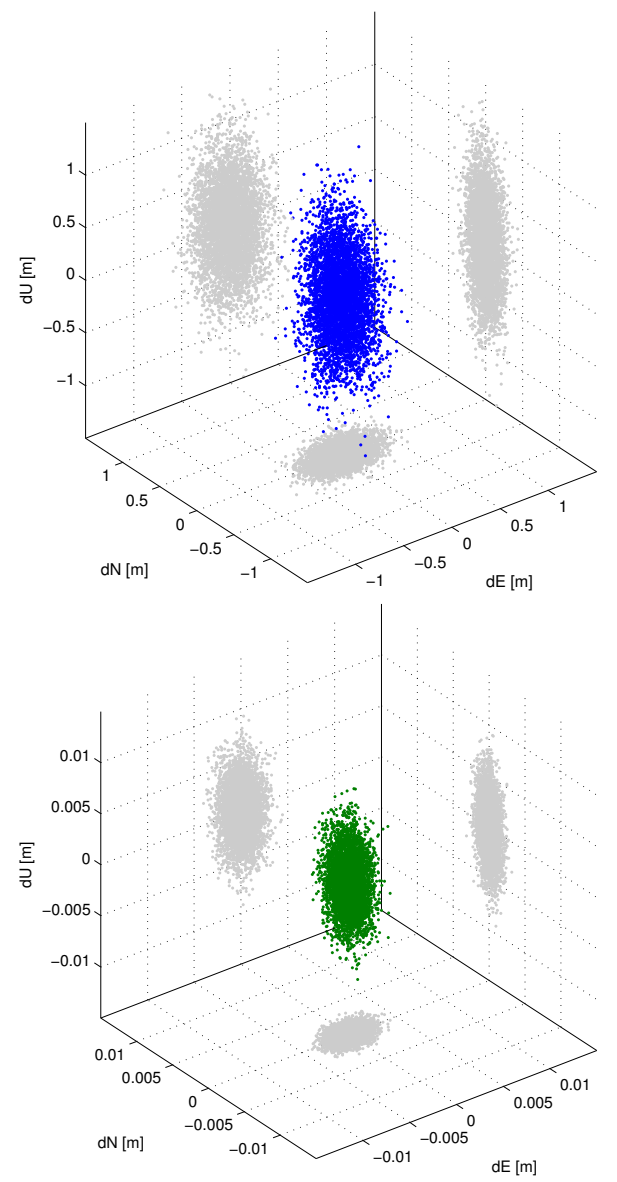

Figure 1: Position errors in East (dE), North $(\mathrm{dN})$ and $\mathrm{Up}(\mathrm{dU})$ direction in meters for ambiguity float solutions (top panel), ambiguity fixed solutions (bottom panel). Note the different scales in the top and bottom panels.

\subsection{Essence of correct integer estimation}

A very high positioning performance can only be guaranteed if the estimated integer ambiguities are correct. It is therefore very important to assess the probability of correct integer estimation. This probability is called the success rate and only if it is very close to 1 , it is possible to rely on the integer solution without further validation. In that case the integer ambiguity solution can be assumed to 
be deterministic, and the VC-matrix of the fixed baseline solution is obtained by application of the propagation law of variances to Eq.(7):

$$
Q_{\breve{b} \breve{b}}=Q_{\hat{b} \hat{b}}-Q_{\hat{b} \hat{a}} Q_{\hat{a} \hat{a}}^{-1} Q_{\hat{a} \hat{b}}
$$

In general $\boldsymbol{Q}_{\breve{b} \breve{b}}<<\boldsymbol{Q}_{\hat{b} \hat{b}}$, since after successful ambiguity fixing the carrier-phase measurements start to act as very precise pseudorange measurements. Figure 1 shows a scatterplot of the float and fixed position errors based on 10,000 solutions with single epoch, dual-frequency GPS for a short baseline; the success rate is equal to 1 . It can be observed that the precision is improved with a factor 100, in agreement with the difference in code and carrier-phase measurement noise.

However, incorrect integer ambiguity estimation may result in the opposite effect in terms of positioning accuracy: rather than a dramatic precision improvement, a wrong ambiguity solution can cause very large position errors, exceeding those of the float solution. This is illustrated in Figure 2, which shows a scatterplot of horizontal float position errors for a case where the ambiguities are fixed correctly in only $93 \%$ of the cases. The corresponding fixed solutions are shown as either red or green dots: red if the ambiguities are fixed incorrectly, green if they are fixed correctly. It can be seen that in all cases where the ambiguities were fixed correctly, the position errors are very small. However, in case of unsuccessful integer estimation the corresponding position errors tend to be of the same size or even much larger than the corresponding float position errors. The figure shows only the horizontal positioning results, for the vertical component the errors can be as large as 8 meters in this example. This clearly shows that the fixed solution should only be used if the success rate is very high. Section 4 presents the Ps-LAMBDA toolbox which allows to evaluate the success rate of integer estimation. 


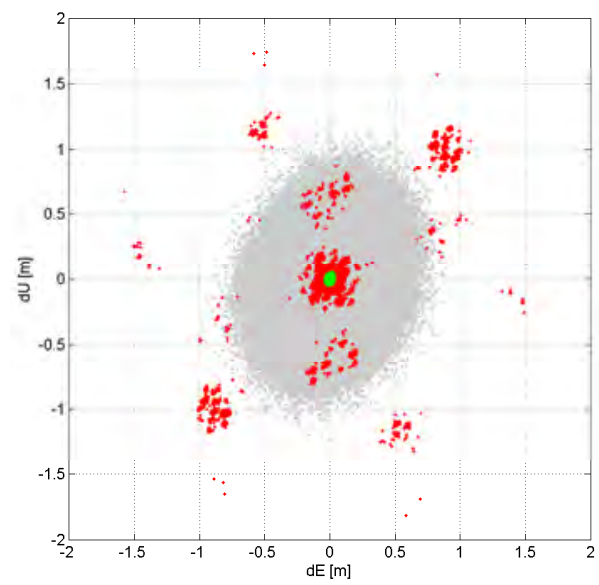

Figure 2: Scatterplot of horizontal position errors in meters for float solution (grey dots) and corresponding fixed solution. In this case, $93 \%$ of the solutions were correctly fixed (green dots), and $7 \%$ was wrongly fixed (red dots).

\section{Admissible integer estimation}

As previously mentioned there are many ways of computing an integer ambiguity vector $\check{\boldsymbol{a}}$ from its real-valued counterpart $\hat{\boldsymbol{a}}$. To each such method belongs a different mapping $\mathcal{I}: \mathbb{R}^{n} \mapsto \mathbb{Z}^{n}$. Due to the discrete nature of $\mathbb{Z}^{n}$, the map $\mathcal{I}$ will not be one-to-one, but instead a many-to-one map. This implies that different real-valued ambiguity vectors will be mapped to the same integer vector. One can therefore assign a subset $\mathcal{P}_{z} \subset \mathbb{R}^{n}$ to each integer vector $z \in \mathbb{Z}^{n}$ :

$$
\mathcal{P}_{z}=\left\{\boldsymbol{x} \in \mathbb{R}^{n} \mid z=\mathcal{I}(\boldsymbol{x})\right\}, \quad z \in \mathbb{Z}^{n}
$$

The subset $\mathcal{P}_{z}$ contains all real-valued ambiguity vectors that will be mapped by $\mathcal{I}$ to the same integer vector $z \in \mathbb{Z}^{n}$. This subset is referred to as the pull-in region of $z$. It is the region in which all ambiguity float solutions are pulled to the same fixed ambiguity vector $z$. 
Using the pull-in regions, one can give an explicit expression for the corresponding integer ambiguity estimator. It reads

$$
\check{\boldsymbol{a}}=\sum_{z \in \mathbb{Z}^{n}} z \mathcal{P}_{z}(\hat{\boldsymbol{a}}), \quad \mathcal{P}_{z}(\hat{\boldsymbol{a}})= \begin{cases}1 & \text { if } \hat{\boldsymbol{a}} \in \mathcal{P}_{z} \\ 0 & \text { otherwise }\end{cases}
$$

Since the pull-in regions define the integer estimator completely, one can define classes of integer estimators by imposing various conditions on the pull-in regions. One such class is referred to as the class of admissible integer estimators. This class was introduced in (Teunissen, 1999b) and it is defined as follows.

\section{Definition}

The integer estimator $\check{\boldsymbol{a}}=\sum_{z \in \mathbb{Z}^{n}} \boldsymbol{z} \mathcal{P}_{z}(\hat{\boldsymbol{a}})$ is said to be admissible if

(i) $\bigcup_{z \in \mathbb{Z}^{n}} \mathcal{P}_{z}=\mathbb{R}^{n}$

(ii) $\quad \operatorname{Int}\left(\mathcal{P}_{z_{1}}\right) \cap \operatorname{Int}\left(\mathcal{P}_{z_{2}}\right)=\emptyset, \quad \forall z_{1}, z_{2} \in Z^{n}, z_{1} \neq z_{2}$

(iii) $\mathcal{P}_{z}=z+\mathcal{P}_{0}, \quad \forall z \in \mathbb{Z}^{n}$

This definition is motivated as follows. The first condition states that the pull-in regions should not leave any gaps and the second that they should not overlap. The absence of gaps is needed in order to be able to map any float solution $\hat{\boldsymbol{a}} \in \mathbb{R}^{n}$ to $\mathbb{Z}^{n}$, while the absence of overlaps is needed to guarantee that the float solution is mapped to just one integer vector. The third and last condition of the definition follows from the requirement that $\mathcal{I}(x+z)=\mathcal{I}(x)+z, \forall x \in \mathbb{R}^{n}, z \in \mathbb{Z}^{n}$. It states that when the float solution is perturbed by $z \in \mathbb{Z}^{n}$, the corresponding integer solution is perturbed by the same amount. This property allows one to apply the integer remove-restore technique: $\mathcal{I}(\hat{\boldsymbol{a}}-\boldsymbol{z})+z=\mathcal{I}(\hat{\boldsymbol{a}})$. 
Integer rounding, integer bootstrapping and integer least squares are all examples of admissible integer estimation methods.

\subsection{Z-transformations}

It will be explained later that it may be useful to apply a so-called $\boldsymbol{Z}$-transformation to the ambiguity parameters. A matrix is called a $\boldsymbol{Z}$-transformation if it is one-toone (i.e. invertible) and integer (Teunissen, 1995a). Such transformations leave the integer nature of the parameters in tact. If a certain integer estimator is $\mathbf{Z}$ invariant it means that if the float solution is $\mathbf{Z}$-transformed, the integer solution transforms accordingly. Hence:

$$
\check{\boldsymbol{z}}=\boldsymbol{Z}^{T} \check{\boldsymbol{a}} \quad \text { if } \quad \hat{z}=\boldsymbol{Z}^{T} \hat{\boldsymbol{a}}
$$

A very useful $\boldsymbol{Z}$-transformation is the decorrelating $\boldsymbol{Z}$-transformation, (Teunissen, 1993, 1994, 1995a,b). It results in a more diagonal VC-matrix:

$$
\boldsymbol{Q}_{\hat{z} \hat{z}}=\boldsymbol{Z}^{T} \boldsymbol{Q}_{\hat{a} \hat{a}} \boldsymbol{Z}
$$

\subsection{Integer rounding}

The simplest way to obtain an integer vector from the real-valued float solution is to round each of the entries of $\hat{\boldsymbol{a}}$ to its nearest integer. The corresponding integer estimator reads

$$
\check{\boldsymbol{a}}_{\mathrm{IR}}=\left(\left[\hat{a}_{1}\right], \cdots,\left[\hat{a}_{n}\right]\right)^{T}
$$

where $[\cdot]$ stands for rounding to the nearest integer.

The pull-in regions for rounding are $n$-dimensional unit cubes centred at the integer grid points:

$$
\mathcal{P}_{z, \mathrm{IR}}=\left\{\boldsymbol{x} \in \mathbb{R}^{n}|| \boldsymbol{c}_{i}^{T}(\boldsymbol{x}-\boldsymbol{z}) \mid \leq \frac{1}{2}, i=1, \ldots, n\right\}, z \in \mathbb{Z}^{n}
$$



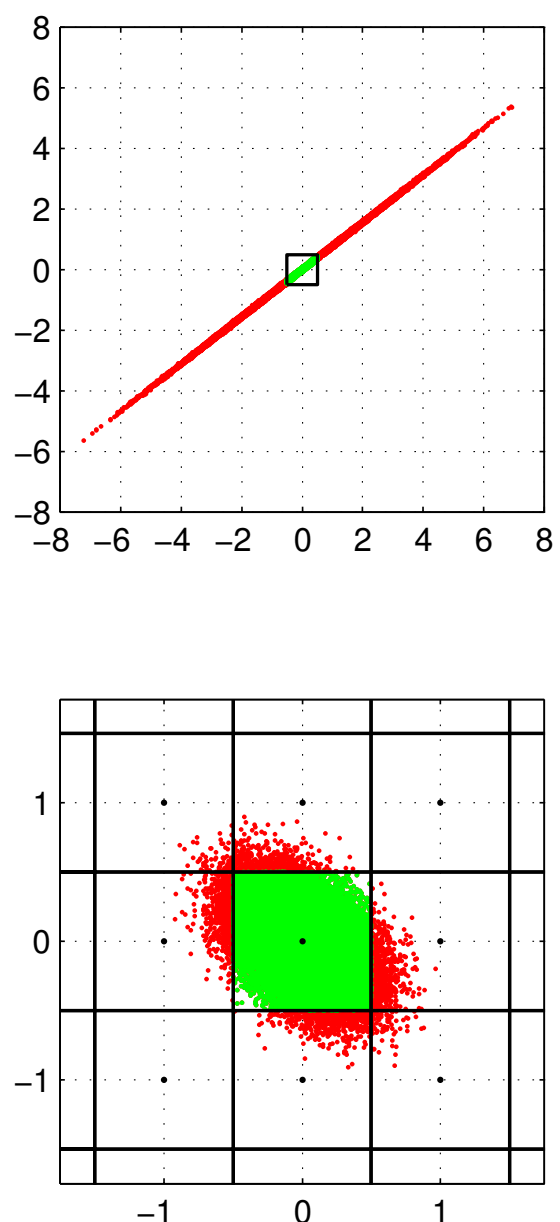

Figure 3: 2D Pull-in regions for integer rounding (IR) and 50,000 float solutions. Top: original ambiguities $\hat{\boldsymbol{a}}$ [cycles]; Bottom: $\boldsymbol{Z}$-decorrelated ambiguities $\hat{z}$ [cycles].

with $\boldsymbol{c}_{i}$ the unit vector have a 1 as its $i$ th entry and 0's otherwise.

In general, the rounding estimator is not $\boldsymbol{Z}$-invariant, i.e. $\check{z}_{\mathrm{IR}} \neq \boldsymbol{Z}^{T} \check{\boldsymbol{a}}_{\mathrm{IR}}$. Only 
samples of float ambiguities for a given VC-matrix $\boldsymbol{Q}_{\hat{a} \hat{a}}$ were simulated; these are shown as the red and green dots. The top panel shows the original float samples (before $\boldsymbol{Z}$-decorrelation), and the pull-in region $\mathcal{P}_{\mathbf{0}, \mathrm{IR}}$, in which all the green samples reside. Hence, for all those samples the $\mathbf{0}$-vector is obtained after rounding. The bottom panel shows the corresponding $\boldsymbol{Z}$-decorrelated float ambiguity samples, as well as the surrounding pull-in regions. In this case, many more float samples reside in $\mathcal{P}_{\mathbf{0} \text {,IR }}: 95 \%$ versus $23 \%$ before $\boldsymbol{Z}$-decorrelation. This shows that the choice for the parameterization of the float ambiguity vector is very important in case of integer rounding.

\subsection{Integer bootstrapping}

The integer bootstrapping (IB) estimator still makes use of integer rounding, but it takes some of the correlation between the ambiguities into account. The IB estimator follows from a sequential least squares adjustment and it is computed as follows. If $n$ ambiguities are available, one starts with the most precise ambiguity. Let the $n$th ambiguity be the most precise one, hence we start with rounding $\hat{a}_{n}$ to the nearest integer. The remaining float ambiguities are corrected by virtue of their correlation with the last ambiguity. Then the last-but-one, but now corrected, real-valued ambiguity estimate is rounded to its nearest integer and all remaining $(n-2)$ ambiguities are then again corrected, but now by virtue of their correlation with this ambiguity. This process is continued until all ambiguities are considered. 

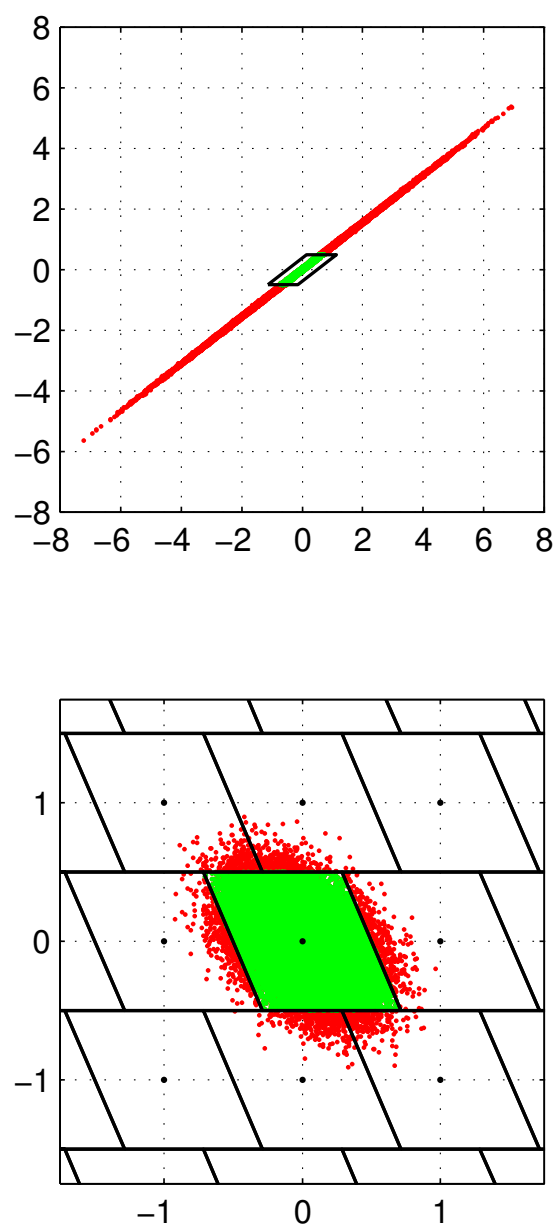

Figure 4: 2D pull-in regions for integer bootstrapping (IB) and 50,000 float solutions. Top: original ambiguities $\hat{\boldsymbol{a}}$ [cycles]; Bottom: $\boldsymbol{Z}$-decorrelated ambiguities $\hat{z}$ [cycles].

The components of the bootstrapped estimator $\check{\boldsymbol{a}}_{\mathrm{IB}}$ are given as

$$
\begin{aligned}
& \check{a}_{n ; \mathrm{IB}}=\left[\hat{a}_{n}\right] \\
& \check{a}_{j ; \mathrm{IB}}=\left[\hat{a}_{j \mid J}\right]=[\hat{a}_{j}-\sum_{i=j+1}^{n} \underbrace{\sigma_{\hat{a}_{j} \hat{a}_{|| I}} \sigma_{\hat{a}_{i \mid l}}^{-2}}_{l_{i, j}}\left(\hat{a}_{i \mid I}-\check{a}_{i ; \mathrm{IB}}\right)], \\
& \forall j=1, \ldots, n-1
\end{aligned}
$$


The short-hand notation $\hat{a}_{i \mid I}$ stands for the $i$ th ambiguity obtained through a conditioning on the previous $I=\{i+1, \ldots, n\}$ sequentially rounded ambiguities. The real-valued sequential least squares solution can be obtained by means of the triangular decomposition of the variance-covariance matrix of the ambiguities: $\boldsymbol{Q}_{\hat{a} \hat{a}}=\boldsymbol{L}^{\boldsymbol{T}} \boldsymbol{D} \boldsymbol{L}$, where $\boldsymbol{L}$ denotes a unit lower triangular matrix with entries $l_{i, j}$ (see Eq.(15)) and $\boldsymbol{D}$ a diagonal matrix with the conditional variances $\sigma_{\hat{a}_{i \mid I}}^{2}$ as its diagonal elements.

The pull-in regions for integer bootstrapping are given as:

$$
\mathcal{P}_{z, \mathrm{IB}}=\left\{\boldsymbol{x} \in \mathbb{R}^{n}|| \boldsymbol{c}_{i}^{T} \boldsymbol{L}^{-T}(\boldsymbol{x}-\boldsymbol{z}) \mid \leq \frac{1}{2}, i=1, \ldots, n\right\}, \boldsymbol{z} \in \mathbb{Z}^{n}
$$

with $\boldsymbol{c}_{i}$ the unit vector have a 1 as its $i$ th entry and 0's otherwise.

Like rounding, bootstrapping suffers as well from a lack of $\boldsymbol{Z}$-invariance, i.e. $\check{\boldsymbol{z}}_{\mathrm{IB}} \neq \boldsymbol{Z}^{T} \check{\boldsymbol{a}}_{\mathrm{IB}}$ if $\hat{\boldsymbol{z}}=\boldsymbol{Z}^{T} \hat{\boldsymbol{a}}$. From Eq.(15) can be seen that changing the order will already result in a different outcome with bootstrapping.

Figure 4 shows a 2D example of the pull-in regions for integer bootstrapping, which in 2D are parallelograms. It can be clearly seen how bootstrapping is affected by the decorrelating $\boldsymbol{Z}$-transformation. Here $96 \%$ of the $\boldsymbol{Z}$-decorrelated float samples resides in $\mathcal{P}_{\mathbf{0}, \mathrm{IB}}$ versus $29 \%$ of the original ambiguity samples.

\subsection{Integer least squares}

When solving the GNSS model of Eq.(3) in a least squares sense, but now with the additional constraint that the ambiguity parameters should be integer-valued, the integer estimator of the second step in the procedure becomes:

$$
\check{\boldsymbol{a}}_{\mathrm{ILS}}=\arg \min _{z \in \mathbb{Z}^{n}}\|\hat{\boldsymbol{a}}-\boldsymbol{z}\|_{\boldsymbol{Q}_{\hat{a} \hat{a}}}^{2}
$$



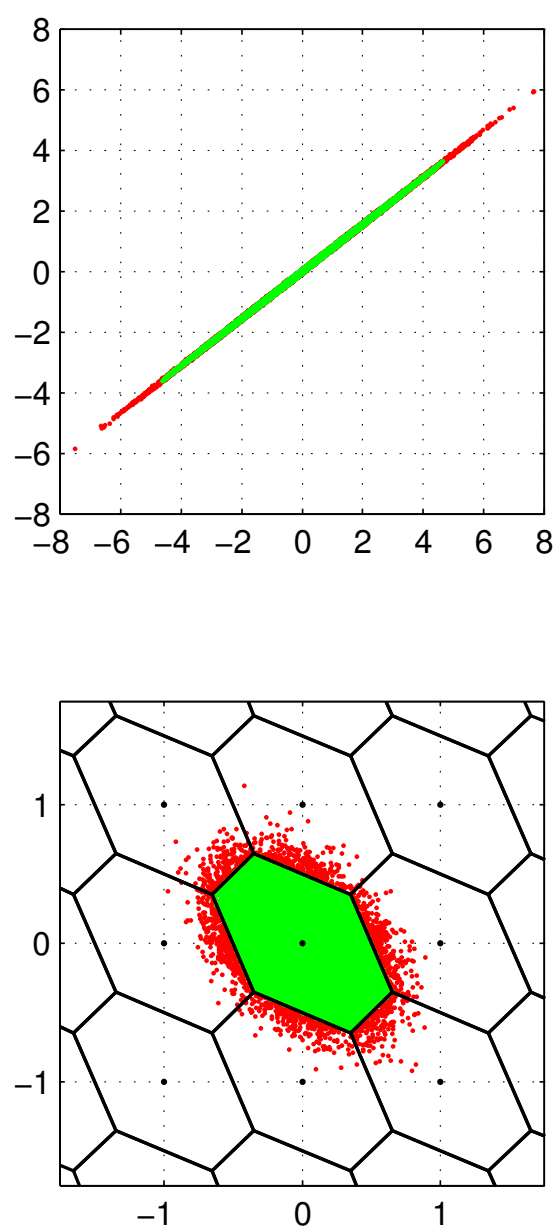

Figure 5: 2D pull-in regions for integer least squares (ILS) and 50,000 float solutions. Top: original ambiguities $\hat{\boldsymbol{a}}$ [cylces]; Bottom: $\boldsymbol{Z}$-decorrelated ambiguities $\hat{z}$ [cylces].

with $\|\cdot\|_{Q}^{2}=(\cdot)^{T} \boldsymbol{Q}^{-1}(\cdot)$. The ILS pull-in region is defined by:

$$
\mathcal{P}_{z, \mathrm{ILS}}=\left\{\boldsymbol{x} \in \mathbb{R}^{n}|| w \mid \leq \frac{1}{2}\|\boldsymbol{u}\|_{\boldsymbol{Q}_{\hat{a} \hat{a}}}, \forall \boldsymbol{u} \in \mathbb{Z}^{n}\right\}
$$

with

$$
w=\frac{\boldsymbol{u}^{T} \boldsymbol{Q}_{\hat{a} \hat{a}}{ }^{-1}(\boldsymbol{x}-\boldsymbol{z})}{\|\boldsymbol{u}\|_{\boldsymbol{Q}_{\hat{a} \hat{a}}}}
$$


Table 2: Percentage of float solutions that is correctly fixed for the three integer estimation methods (corresponding to Figures 3 to 5).

\begin{tabular}{l|ccc} 
& IR & IB & ILS \\
\hline Original ambiguities $\hat{\boldsymbol{a}}$ & 23 & 29 & 97 \\
$\boldsymbol{Z}$-decorrelated ambiguities $\hat{\boldsymbol{z}}$ & 95 & 96 & 97
\end{tabular}

the orthogonal projection of $(\boldsymbol{x}-\boldsymbol{z})$ onto the direction vector $\boldsymbol{u}$. Hence, $\mathcal{P}_{z \text { ILS }}$ is the intersection of banded subsets centered at $\boldsymbol{z}$ and having width $\|\boldsymbol{u}\|_{\boldsymbol{Q}_{\hat{a} \hat{a}}}$.

In contrast to integer rounding and integer bootstrapping, the ILS estimator is $\boldsymbol{Z}$-invariant: $\check{\boldsymbol{z}}_{\mathrm{ILS}}=\boldsymbol{Z}^{T} \check{\boldsymbol{a}}_{\mathrm{ILS}}$ if $\hat{\boldsymbol{z}}=\boldsymbol{Z}^{T} \hat{\boldsymbol{a}}$.

Figure 5 shows an example of the 2D pull-in regions for integer least squares. For the original VC-matrix $\boldsymbol{Q}_{\hat{a} \hat{a}}$ (top panel) the ILS pull-in region follows the distribution of the float samples much better than in case of rounding and bootstrapping, compare with the corresponding Figures 3 and 4 . Due to the $\boldsymbol{Z}$-invariance the percentage of float samples in $\mathcal{P}_{\mathbf{0}, \text { ILS }}$ (the green dots) is $97 \%$ both for the original and $\boldsymbol{Z}$-decorrelated ambiguities. The percentages for all three integer estimators are summarized in Table 2.

An integer search is needed to determine $\check{\boldsymbol{a}}_{\mathrm{ILS}}$. The ILS procedure is efficiently mechanized in the LAMBDA (Least squares AMBiguity Decorrelation Adjustment) method. A key element of the LAMBDA method is the decorrelating $Z$-transformation, see Section 3.1, which results in largely reduced search times. For more information on the LAMBDA method and its wide-spread applications see e.g. (Teunissen, 1993, 1995b; Li and Teunissen, 2011; Chang et al., 2005; De Jonge and Tiberius, 1996; Hofmann-Wellenhof et al., 2001; Teunissen and Kleusberg, 1998; Leick, 2004; Strang and Borre, 1997; Misra and Enge, 2001). 


\section{Success Rate: definition and evaluation tool}

In Section 2.3 the essence of correct integer estimation was described. It is thus important to have means available to evaluate the ambiguity success rate, i.e., the probability of correct integer estimation, $P_{s}$. This success rate is equal to the probability that $\hat{\boldsymbol{a}}$ resides in the correct pull-in region $\mathcal{P}_{\boldsymbol{a}}$ with $\boldsymbol{a}$ the true but unknown ambiguity vector:

$$
P_{s}=P(\check{\boldsymbol{a}}=\boldsymbol{a})=P\left(\hat{\boldsymbol{a}} \in \mathcal{P}_{\boldsymbol{a}}\right)=\int_{\mathcal{P}_{\boldsymbol{a}}} f_{\hat{\boldsymbol{a}}}(\boldsymbol{x} \mid \boldsymbol{a}) d \boldsymbol{x}
$$

The probability density function (PDF) of the float ambiguities, $f_{\hat{a}}(\boldsymbol{x} \mid \boldsymbol{a})$, is assumed to be the normal PDF with mean $\boldsymbol{a}$ :

$$
f_{\hat{a}}(\boldsymbol{x} \mid \boldsymbol{a})=\frac{1}{\sqrt{\operatorname{det}\left(2 \pi Q_{\hat{a} \hat{a})}\right.}} \exp \left\{-\frac{1}{2}(\boldsymbol{x}-\boldsymbol{a})^{T} Q_{\hat{\boldsymbol{a}} \hat{\boldsymbol{a}}}^{-1}(\boldsymbol{x}-\boldsymbol{a})\right\}
$$

As the pull-in regions of the integer estimators are integer-translation invariant, the success rate can also be evaluated as:

$$
P_{s}=\int_{\mathcal{P}_{\mathbf{0}}} f_{\hat{\boldsymbol{a}}}(\boldsymbol{x} \mid \mathbf{0}) d \boldsymbol{x}
$$

An illustration is given in Figure 6 for the ILS estimator: in the top panel the PDF of a 2D float ambiguity vector is shown, with the corresponding ILS pullin regions underneath. The bottom panel shows the probability masses for each integer grid point, equal to the integral of the PDF over the corresponding pull-in regions. In this case, the success rate is equal to the probability mass at $\left[\begin{array}{ll}0 & 0\end{array}\right]^{T}$.

From the definition (22) it follows that the success rate depends on the integer estimation method (IR, IB or ILS) as well as on the float ambiguity precision captured by VC-matrix $\boldsymbol{Q}_{\hat{a} \hat{a}}$. From Eq.(4) follows that:

$$
Q_{\hat{a} \hat{a}}=\left(A^{T} Q_{y y}^{-1} A-A^{T} Q_{y y}^{-1} B\left(B^{T} Q_{y y}^{-1} B\right)^{-1} B^{T} Q_{y y}^{-1} A\right)^{-1}
$$



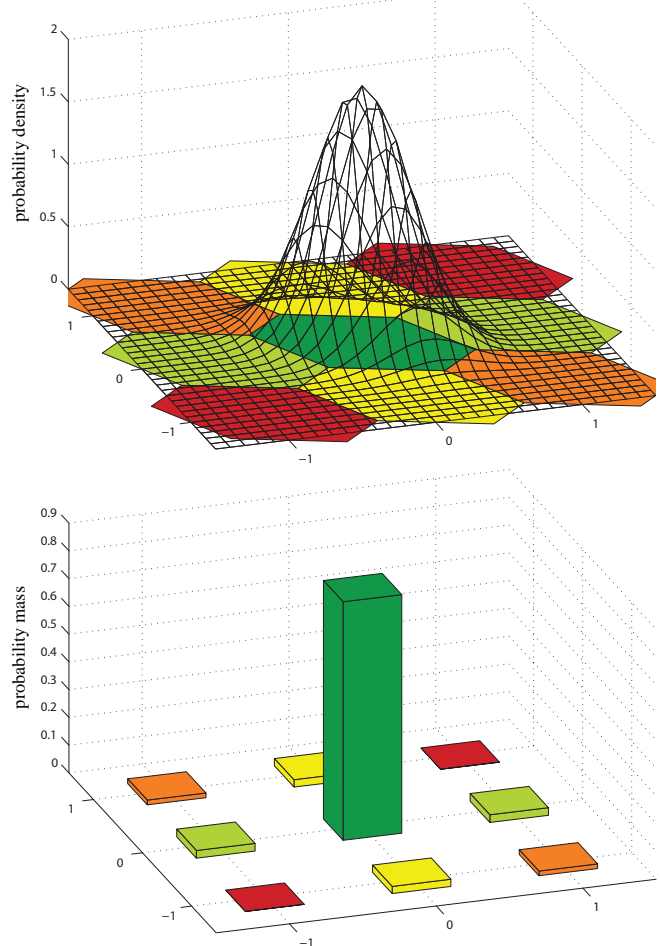

Figure 6: Top: PDF and 2D pull-in regions for ILS. Bottom: corresponding probability mass function. 


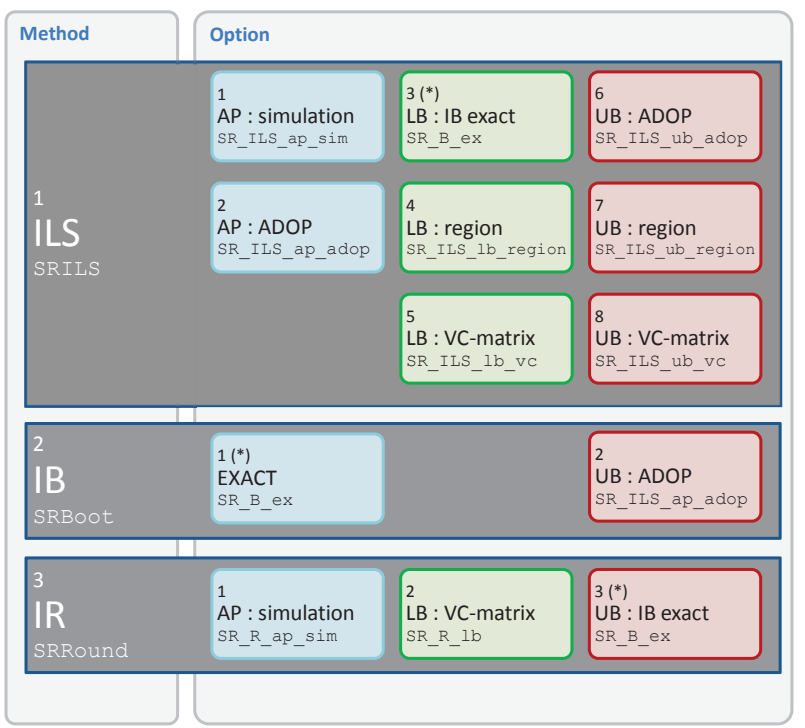

Figure 7: Ps-LAMBDA: overview of available methods and options in routine SuccessRate. Default option is indicated with $(*)$. Names of underlying routines are shown as well. AP = approximation (blue), LB = lower bound (green), UB = upper bound (red). 
From this it can be seen that the following factors drive the ambiguity float precision:

- receiver-satellite geometry (depends on time and location, as well as on which GNSS is used) $\rightarrow \boldsymbol{B}, \boldsymbol{Q}_{y y}$

- measurement noise (depends on GNSS signal and receiver quality) $\rightarrow \boldsymbol{Q}_{y y}$

- assumptions on atmospheric delays (depends on atmosphere models and/or between-receiver baseline length) $\rightarrow \boldsymbol{B}, \boldsymbol{Q}_{\boldsymbol{y} y}$

- frequencies used (depends on GNSS and receiver) $\rightarrow \boldsymbol{A}$

- number of observation epochs $\rightarrow \boldsymbol{B}$

Note that the satellite geometry only affects $\boldsymbol{Q}_{\boldsymbol{y} y}$ if elevation-dependent weighting is applied to the observations. The influence of the atmosphere delays depends on whether the delays are estimated and thus included as unknown parameters in $\boldsymbol{b}$, and what uncertainty is assigned to the corrections if applied.

Since the success rate can be computed once the float ambiguity VC-matrix $\boldsymbol{Q}_{\hat{a} \hat{a}}$ is known, it can be computed without the need for actual data. As such, the success rate can be used as a very important performance measure for:

- planning purposes (design computations): what is the performance to be expected given a certain measurement set-up at a given time and location;

- deciding whether or not to fix the ambiguities to the integer estimates during the actual data processing (in real-time or post-processing mode); 
- research purposes, e.g. to study the impact of receiver noise characteristics, availability of more signals / satellites, baseline length, etcetera.

As mentioned, the success rates also depend on the selected integer estimation method, since the pull-in region is different for IR, IB and ILS. In (Teunissen, 1999a) it was proven that:

$$
P\left(\check{\boldsymbol{a}}_{\mathrm{IR}}=\boldsymbol{a}\right) \leq P\left(\check{\boldsymbol{a}}_{\mathrm{IB}}=\boldsymbol{a}\right) \leq P\left(\check{\boldsymbol{a}}_{\mathrm{ILS}}=\boldsymbol{a}\right)
$$

The ordering is thus the same as the ordering in terms of complexity, since IR is the simplest and ILS the most complex method. This means that if IR or IB provides a very sharp lower bound, a user could decide to use the simpler integer estimation method if their success rate is close to 1 and still obtain (close to) optimal performance.

The success rate cannot be evaluated exactly in all cases due to the complex integration over the pull-in region. It is of course important to be able to have good approximations of the success rate in case exact evaluation is not feasible. A lower bound is an approximation of the success rate, which is guaranteed to be smaller than or equal to the actual success rate. As such it is particularly useful. However, if the lower bound is not tight, this may result in a unnecessarily high rejection rate as the success rate is deemed too low. An upper bound can be useful as well, especially in combination with a lower bound, since it then tells the user in which range the success rate will be. If the upper bound is below a user-defined threshold, one cannot expect ambiguity resolution to be successful. In addition, for IR and IB it may be useful to have an upper bound which is invariant for the class of admissible ambiguity transformations. 
Different approximations and bounds were proposed in literature, an evaluation of some of the bounds was made in (Verhagen, 2005; Thomsen, 2000). All bounds and approximations are now implemented in a newly developed Matlab toolbox, called Ps-LAMBDA. Figure 7 gives an overview of the structure. The main routine is SuccessRate which needs as input:

Qa the VC-matrix of the float ambiguities $\boldsymbol{Q}_{\hat{a} \hat{a}}$

method $1=\mathrm{ILS}[$ DEFAULT], $2=\mathrm{IB}, 3=\mathrm{IR}$

option the approximation / bound to compute (see Figure 7)

decor $1=$ decorrelation [DEFAULT]

$0=$ no decorrelation

nsamp number of samples only used for simulation-based approximation

The choice for decor is only relevant for IR and IB, since these estimators are not $\boldsymbol{Z}$-invariant. Decorrelation is always applied for ILS to ensure computational efficiency.

The toolbox also includes a Graphical User Interface which allows the user to select an input file which contains the VC-matrix $\boldsymbol{Q}_{\hat{a} \hat{a}}$ and to compute all the desired bounds and approximations for different integer estimation methods simultaneously.

In the next section the bounds and approximations for each of the three estimators are presented. The performance of the bounds and approximations will be assessed for different GNSS models, where the different factors affecting the float ambiguity precision are varied as shown in Table 3. An exponential elevationdependent weighting is applied (more noise is assumed for observations from 
Table 3: Measurement scenarios used in Section 5 (standard deviations apply to zenith direction).

\begin{tabular}{ll}
\hline system & $\begin{array}{l}\text { GPS - } \\
\text { combined GPS+Galileo }\end{array}$ \\
\hline times & 49 different epochs \\
\hline frequencies & L5 - L1+L5 - \\
& L1+L5+L2/E5b \\
\hline standard deviations of & code: $15 \mathrm{~cm}$ \\
undifferenced observations & phase: $1 \mathrm{~mm}$ \\
\hline VC-matrix scale factors & $0.25-0.5-1-2-4$ \\
\hline standard deviation of & \\
ionosphere corrections & $5-15 \mathrm{~mm}$ \\
\hline
\end{tabular}

low-elevation satellites) to the standard deviations of the observations and of the ionosphere corrections. The scale factors applied to the VC-matrix $\boldsymbol{Q}_{\hat{a} \hat{a}}$ can either be interpreted as representing a different number of epochs, or a different measurement precision due to different receiver quality.

\section{Success Rate: bounds and approximations}

\subsection{Approximation based Monte Carlo simulations}

The success rate of integer estimation can be approximated by means of Monte Carlo simulation. The procedure is as follows. It is assumed that the float solution is normally distributed $\hat{\boldsymbol{a}} \sim N\left(\boldsymbol{a}, \boldsymbol{Q}_{\hat{a} \hat{a}}\right)$, and thus the distribution is symmetric about the mean $\boldsymbol{a}$. Hence, we may shift the distribution over $\boldsymbol{a}$ and draw samples from the distribution $N\left(\mathbf{0}, \boldsymbol{Q}_{\hat{a} \hat{a}}\right)$. 

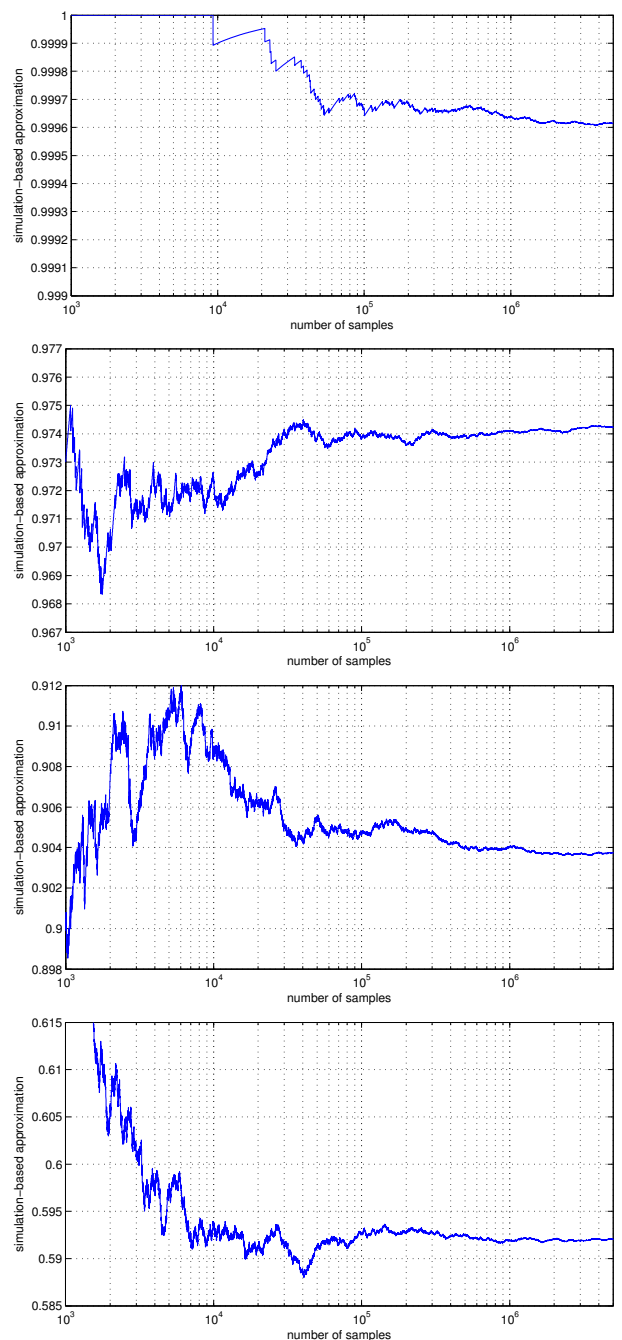

Figure 8: Examples of simulation-based success rate as function of number of samples. Each panel shows the results for a different GNSS model.

The first step is to use a random generator to generate $n$ independent samples from the univariate standard normal distribution $N(0,1)$, and then collect these in a vector $\boldsymbol{s}$. This vector is transformed by means of $\hat{\boldsymbol{a}}=\boldsymbol{G s}$, with $\boldsymbol{G}$ equal to the 
this sample is used as input for integer estimation. If the output of this estimator equals the null vector, then it is correct, otherwise it is incorrect. This process can be repeated an $N$ number of times, and one can count how many times the null vector is obtained as a solution, say $N_{s}$ times. The approximation of the success rate follows then as:

$$
P_{s} \approx \frac{N_{s}}{N}
$$

In order to get good approximations, the number of samples $N$ must be sufficiently large (Teunissen, 1998a). The disadvantage is that it may be very time-consuming to evaluate Eq.(25), especially in case of ILS, since for each sample an integer search is required.

The concept of approximating the success rate based on simulations was already applied in Sections 3.2-3.4, see Table 2.

Figure 8 shows for four GNSS models how the approximation performs depending on the number of samples used (similar results were obtained for many other GNSS positioning models). It follows that at least $10^{5}$ samples should be used to get a good approximation. At the same time it can be seen that using more samples generally only has a small effect, in the order of $10^{-3}$, especially in cases where the success rate is close to 1 . With $10^{6}$ samples the approximation will be very close to the true value. In the remainder of this contribution the simulationbased success rates will be compared to other bounds and approximations. The number of samples used is $10^{6}$.

Ps-LAMBDA allows to evaluate the simulation-based success rates for IR and ILS (option 1 in Figure 7), where the user may specify the number of samples to be used. The option is not available for IB, as its success rate can be evaluated exactly, as will be shown in Section 5.3. 


\subsection{Integer Rounding success rates}

The $n$-fold integral over the IR pull-in region defined in (14) is difficult to evaluate. Only if the VC-matrix $\boldsymbol{Q}_{\hat{a} \hat{a}}$ is diagonal will the success rate become equal to the $n$-fold product of the univariate success rates. In (Teunissen, 1998b) it was shown that this also provides a lower bound in case $\boldsymbol{Q}_{\hat{a} \hat{a}}$ is not diagonal:

$$
P_{s, \mathrm{IR}}=P\left(\check{\boldsymbol{a}}_{\mathrm{IR}}=\boldsymbol{a}\right) \geq \prod_{i=1}^{n}\left(2 \Phi\left(\frac{1}{2 \sigma_{\hat{a}_{i}}}\right)-1\right)
$$

with $\Phi(x)$ the cumulative normal distribution function:

$$
\Phi(x)=\frac{1}{\sqrt{2 \pi}} \int_{-\infty}^{x} \exp \left\{-\frac{1}{2} t^{2}\right\} d t
$$

In Section 3.2 it was mentioned that IR is not $\boldsymbol{Z}$-invariant. This holds for the IR success rates as well, since the pull-in regions are unaffected by a $\boldsymbol{Z}$ transformation, while the distribution of the transformed ambiguities is changed to $\hat{z} \sim N\left(\boldsymbol{Z}^{T} \boldsymbol{a}, \boldsymbol{Q}_{\hat{z} \hat{z}}\right)$. If IR is applied to the $\boldsymbol{Z}$-decorrelated ambiguities, the success rate will increase due to the improved precision of the decorrelated ambiguities, i.e.

$$
P\left(\check{z}_{\mathrm{IR}}=\boldsymbol{z}\right) \geq P\left(\check{\boldsymbol{a}}_{\mathrm{IR}}=\boldsymbol{a}\right)
$$

According to Eq.(24), IB will always result in a success rate higher than or equal to the IR success rate if the same parameterization of the float ambiguities is used. Hence, the IB success rate can be used as an upper bound for IR. In the next subsection it will be shown that the IB success rate can in fact be evaluated exactly.

Figure 9 shows the lower bound and upper bound versus the actual IR success rates (all for the decorrelated ambiguities). It can be seen that the lower bound is very tight, whereas the upper bound based on the IB success rate is not as 


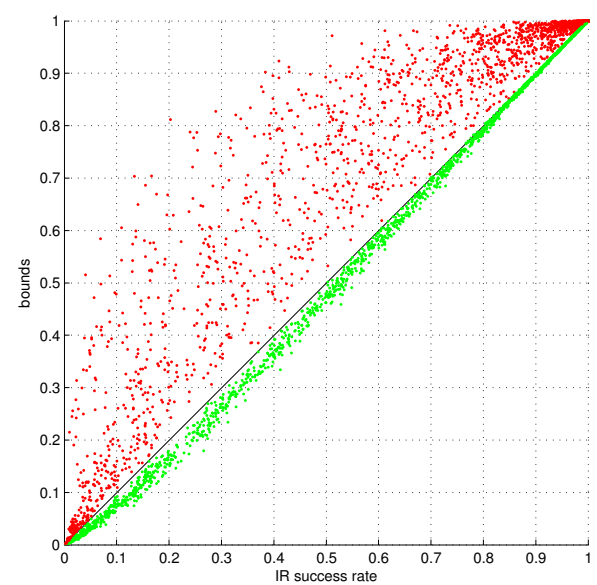

Figure 9: IR success rates: upper bound based on IB (red) and lower bound based on diagonal VC-matrix (green) versus the actual IR success rate for the models from Table 3.

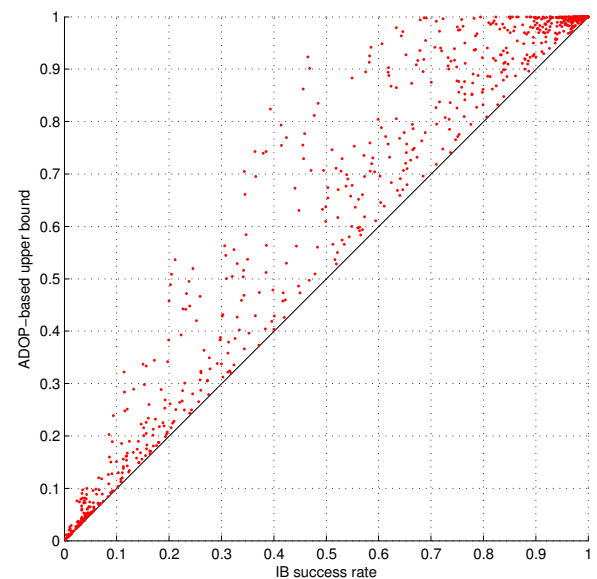

Figure 10: IB success rates: ADOP-based upper bound versus the exact IB success rate for the models from Table 3. integer rounding. 


\subsection{Integer Bootstrapping success rates}

In case of bootstrapping the success rate can be evaluated exactly using (Teunissen, 1998b):

$$
P_{s, \mathrm{IB}}=P\left(\check{\boldsymbol{a}}_{\mathrm{IB}}=\boldsymbol{a}\right)=\prod_{i=1}^{n}\left(2 \Phi\left(\frac{1}{2 \sigma_{\hat{a}_{i \mid}}}\right)-1\right)
$$

The IB success rate is not $\boldsymbol{Z}$-invariant. Bootstrapping may perform close to optimal if applied to the decorrelated ambiguities $\hat{z}$, (Teunissen, 1998b; Verhagen, 2005), and as with rounding we have:

$$
P\left(\check{z}_{\mathrm{IB}}=\boldsymbol{z}\right) \geq P\left(\check{\boldsymbol{a}}_{\mathrm{IB}}=\boldsymbol{a}\right)
$$

For bootstrapping we thus have an exact and easy-to-compute formula for the success rate. Still it can be useful to have an upper bound, which is $\boldsymbol{Z}$-invariant, since if this upper bound is too small, it can be immediately concluded that neither bootstrapping, nor rounding, will be successful for any parameterization of the ambiguities. In Teunissen (2000) it was proven that such an upper bound is given by:

$$
P_{s, \mathrm{IB}} \leq\left(2 \Phi\left(\frac{1}{2 A D O P}\right)-1\right)^{n}
$$

with ADOP being the Ambiguity Dilution of Precision given by:

$$
A D O P=\sqrt{\operatorname{det}\left(\boldsymbol{Q}_{\hat{a} \hat{a}}\right)^{\frac{1}{n}}}
$$

with units of cycles. The ADOP is a diagnostic that captures the main characteristics of the ambiguity precision. It was introduced in (Teunissen, 1997), described and analyzed in (Teunissen and Odijk, 1997; Odijk and Teunissen, 2008) and is widely used, see the introduction of (Odijk and Teunissen, 2008). The ADOP is invariant for the class of admissible ambiguity transformation, i.e. 
$\operatorname{det}\left(\boldsymbol{Q}_{\hat{a} \hat{a}}\right)=\operatorname{det}\left(\boldsymbol{Q}_{\hat{z} \hat{z}}\right)$. When the ambiguities are completely decorrelated, the ADOP equals the geometric mean of the standard deviations of the ambiguities, hence it can be considered as a measure of the average ambiguity precision.

Figure 10 shows that the upper bound is in these cases often significantly higher than the exact success rate $P\left(\check{z}_{\mathrm{IB}}=z\right)$. Better bounding performance is obtained for lower dimensions $n$, which is due to the replacement of the $n$ conditional standard deviations in Eq.(28) by a single value equal to ADOP.

\subsection{Integer Least Squares success rates}

Due to the complex geometry of the ILS pull-in region defined in Eq.(18), the multivariate integral in Eq.(22) can only be evaluated by using Monte Carlo simulation. In addition, several lower and upper bounds of the ILS success rate have been proposed. They will all be presented here.

\section{Bounds and approximations based on IB and ADOP}

Teunissen (1999a) proved that the ILS estimator is optimal, in the sense that it gives the maximum success rate. Furthermore, it was already mentioned that IB may perform close to optimal if applied to decorrelated ambiguities. Therefore the corresponding IB success rate can be used as a lower bound for the ILS success rate:

$$
P_{s, \mathrm{ILS}}=P\left(\check{\boldsymbol{a}}_{\mathrm{ILS}}=\boldsymbol{a}\right) \geq \prod_{i=1}^{n}\left(2 \Phi\left(\frac{1}{2 \sigma_{\hat{z}_{i \mid I}}}\right)-1\right)
$$

The conditional standard deviations $\sigma_{\hat{z}_{i \mid l}}$ of the decorrelated ambiguities must be used (see Eq.(29)).

Consequently, the invariant upper bound of the IB success rate from Eq.(30) may serve as an approximation of the ILS success rate. 


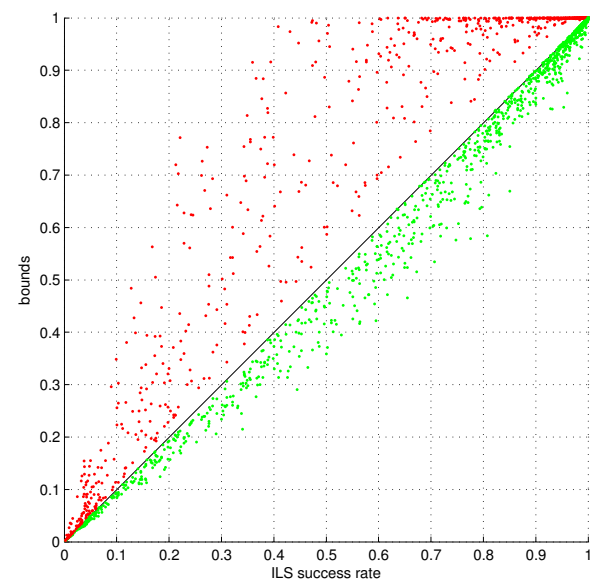

Figure 11: ILS success rates: lower bound based on IB (green) and upper bound based on ADOP (red) versus the actual ILS success rate for the models from Table 3.

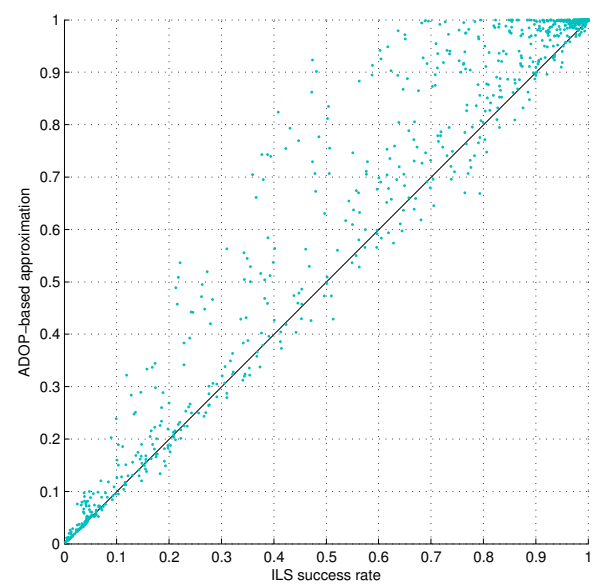

Figure 12: ILS success rates: ADOP-based approximation versus the actual ILS success rate for the models from Table 3.

Furthermore, an upper bound for the ILS success rate based on the ADOP can be given as:

$$
P_{s, \mathrm{ILS}} \leq P\left(\chi^{2}(n, 0) \leq \frac{c_{n}}{A D O P^{2}}\right)
$$


with

$$
c_{n}=\frac{\left(\frac{n}{2} \Gamma\left(\frac{n}{2}\right)\right)^{\frac{2}{n}}}{\pi}
$$

This bound was introduced in Hassibi and Boyd (1998), while the proof was given in Teunissen (2000).

Figure 11 shows how the IB success rate performs as a lower bound for ILS. In practice, the IB success rate is commonly used as the best known lower bound, and these results confirm that especially if the success rate is high, this is indeed the case. At the same time, it can be seen how ILS may still significantly outperform IB for lower success rates.

Figure 11 shows that for these cases the ADOP-based upper bound often gives a too optimistic value compared to the actual success rate. As is shown later, however, the bounding performance improves for lower dimensions (cf. Figure 17).

A similar conclusion can be given for the ADOP-based approximation of the ILS success rate as shown in Figure 12. Only in some of these cases can it be used as a coarse approximation. The approximation improves in case of lower dimensions (cf. Figure 17).

\section{Bounds based on bounding the integration region}

In (Teunissen, 1998a) lower and upper bounds for the ILS success rate were obtained by bounding the integration region. Obviously, a lower bound is obtained if the integration region is chosen such that it is completely contained by the pullin region, and an upper bound is obtained if the integration region is chosen such that it completely contains the pull-in region. The integration region can then be chosen such that the integral is easy-to-evaluate. In ibid the integration region for 

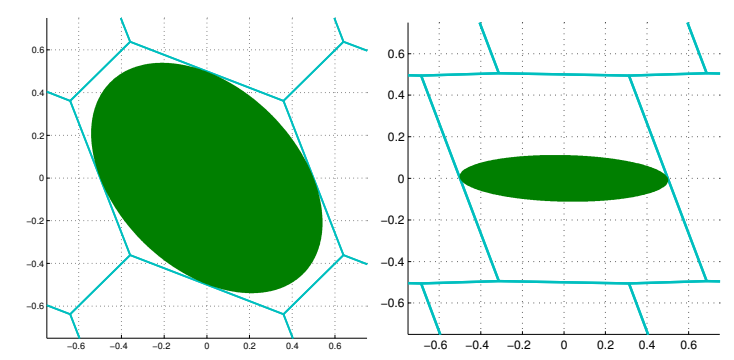

Figure 13: Two examples of the ellipsoidal region (green) contained by the pull-in region $\mathcal{P}_{\mathbf{0} \text {,ILS }}$ (different shape of pull-in regions is due to different VC-matrices $\boldsymbol{Q}_{\hat{z} \hat{z}}$ ).

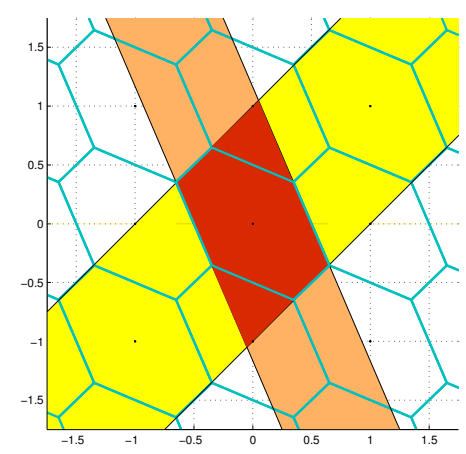

Figure 14: Integration region (red) containing $\mathcal{P}_{\mathbf{0}, \text { ILS }}$ and defined by the intersection of two banded subsets.

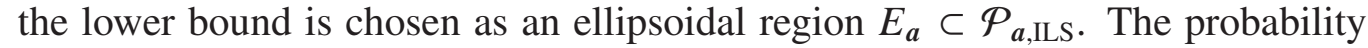
$P\left(\hat{\boldsymbol{a}} \in E_{\boldsymbol{a}}\right)$ can be evaluated based on the $\chi^{2}$-distribution:

$$
P_{s, \mathrm{ILS}} \geq P\left(\hat{\boldsymbol{a}} \in E_{\boldsymbol{a}}\right)=P\left(\chi^{2}(n, 0) \leq \frac{1}{4} \min _{\boldsymbol{u} \in \mathbb{Z}^{n} \backslash\{0\}}\|\boldsymbol{u}\|_{\boldsymbol{Q}_{\hat{a} \hat{\boldsymbol{a}}}}^{2}\right)
$$

The concept is illustrated in Figure 13 for two different pull-in regions, corresponding to different VC-matrices $\boldsymbol{Q}_{\hat{z} \hat{z}}$.

The upper bound can thus be obtained by defining a region $U_{\boldsymbol{a}} \supset \mathcal{P}_{\boldsymbol{a} \text {,ILS }}$. Given the definition of the ILS pull-in region $\mathcal{P}_{a \text {,ILS }}$ in Eq.(18), it follows that any finite intersection of $p<n$ banded subsets defined by $w$ of Eq.(19) will enclose $\mathcal{P}_{a \text {,ILS. }}$. The idea is illustrated in Figure 14 for the $2 \mathrm{D}$ case where $U_{\boldsymbol{a}}$ is chosen as the 
intersection of two banded subsets. The probability $P\left(\hat{\boldsymbol{a}} \in U_{\boldsymbol{a}}\right)$, however, cannot be evaluated exactly either, but can be bounded from above to obtain (Teunissen, 1998a):

$$
P_{s, \mathrm{ILS}} \leq P\left(\hat{\boldsymbol{a}} \in U_{\boldsymbol{a}}\right) \leq \prod_{i=1}^{p}\left(2 \Phi\left(\frac{1}{2 \sigma_{v_{i||}}}\right)-1\right)
$$

with the conditional standard deviation $\sigma_{v_{i \mid I}}$ of vector $v$. These are equal to the square root of the diagonal elements of $\boldsymbol{D}$ from the $\boldsymbol{L}^{T} \boldsymbol{D} \boldsymbol{L}$-decomposition of $\boldsymbol{Q}_{v v}$ with its elements given by:

$$
\sigma_{v_{i} v_{j}}=\frac{\boldsymbol{u}_{i}^{T} \boldsymbol{Q}_{\hat{a} \hat{a}}^{-1} \boldsymbol{u}_{j}}{\left\|\boldsymbol{u}_{i}\right\|_{Q_{\hat{a} \hat{a}}}\left\|\boldsymbol{u}_{j}\right\|_{Q_{\hat{a} \hat{a}}}}, \quad \boldsymbol{u}_{i}, \boldsymbol{u}_{j} \in \mathbb{Z}^{n}
$$

where the $\boldsymbol{u}_{i}, i=1, \ldots, p$ need to be linearly independent. How to evaluate this upper bound is described in (Teunissen, 1998a; Verhagen, 2005). Note that in the higher dimensional case many subsets are necessary to obtain a tight upper bound, and selection of the subset is rather complicated. In addition, it is computationally demanding, since the determination of the subset involves the evaluation of many integer candidates to be obtained with LAMBDA.

Kondo (2003) presented a lower bound of the ILS success rate by replacing the conditional standard deviation $\sigma_{v_{i \mid l}}$ in Eq.(35) by the unconditional standard deviation $\sigma_{v_{i}}$. In Verhagen (2005) it was explained that this is only guaranteed to be a lower bound under certain conditions, which are difficult to fulfill.

Figure 15 shows the lower and upper bound of the ILS success rate based on bounding the integration region. It can be seen that the upper bound performs reasonably well, whereas the lower bound is generally not tight at all - it will be close to zero unless the success rate is very close to 1 . The bad performance can be explained based on the 2D example on the right-hand side of Figure 13: the ellipsoidal region may leave a large part of the ILS pull-in region uncovered. This 


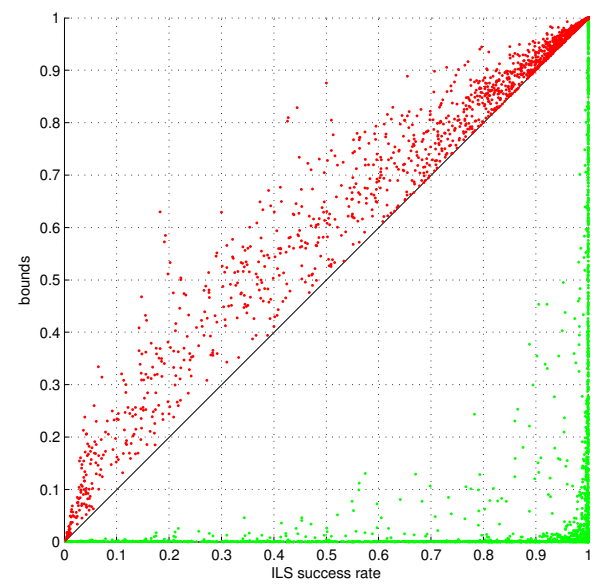

Figure 15: ILS success rates: lower and upper bounds based on bounding the integration region versus the actual ILS success rate for the models from Table 3.

will be the case when there is a large variation in the variances $\sigma_{\hat{z}_{\hat{i}} \hat{z}_{i}}$ (making the ellipsoidal region elongated).

\section{Bounds based on bounding the VC-matrix}

It is also possible to obtain a lower and an upper bound by bounding the actual VC-matrix from above and below by diagonal matrices, and then to compute the probability of correct integer estimation belonging to these diagonal matrices, (Teunissen, 1998a). The simplest way is to bound the actual VC-matrix with:

$$
\lambda_{\min } \boldsymbol{I}_{n} \leq \boldsymbol{Q}_{\hat{z} \hat{z}} \leq \lambda_{\max } \boldsymbol{I}_{n}
$$

where $\lambda_{\min }$ and $\lambda_{\max }$ are the minimum and maximum eigenvalues of $\boldsymbol{Q}_{\hat{z} \hat{z}}$, and $\boldsymbol{I}_{n}$ is an identity matrix of order $n$. The ILS success rate bounds follow as:

$$
\left(2 \Phi\left(\frac{1}{2 \sqrt{\lambda_{\max }}}\right)-1\right)^{n} \leq P_{s, \mathrm{ILS}} \leq\left(2 \Phi\left(\frac{1}{2 \sqrt{\lambda_{\min }}}\right)-1\right)^{n}
$$

Figure 16 shows the lower and upper bound of the ILS success rate based on bounding the VC-matrix. It can be seen that both bounds perform poorly. 


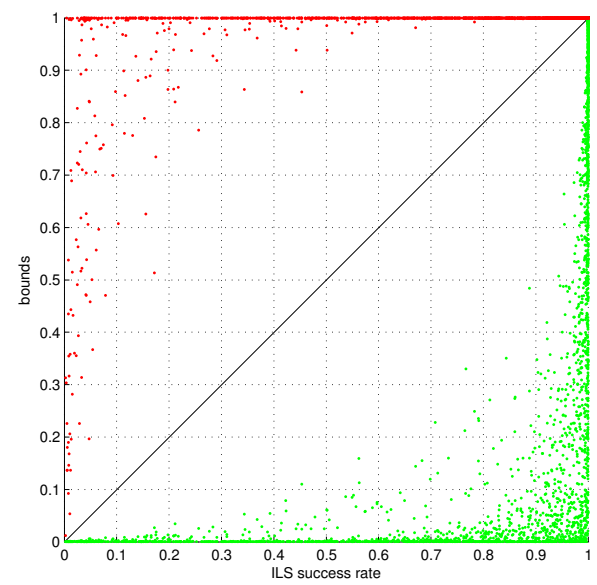

Figure 16: ILS success rates: lower and upper bounds based on bounding the VC-matrix versus the actual ILS success rate for the models from Table 3.

Similarly as with the ADOP-based approximation of the ILS success rate, this is especially true for large $n$ due to the replacement of the $n$ conditional standard deviations in Eq.(32) by the square root of the minimum or maximum eigenvalue, respectively.

\section{Examples with other models}

So far, the performance of the success rate bounds and approximations was analyzed based on the linearized DD GNSS model parameterized in terms of the baseline unknowns. However, it is also possible to use the observation equations (1) directly, and hence parameterize the DD model in terms of the satellite-receiver ranges. This model is referred to as the geometry-free model, and is used for example for integrity monitoring or as a first step in the data processing. Here, we will show an example based on a dual-frequency GNSS model for one satellitereceiver pair (i.e. one DD code and phase observation per frequency). The GPS L1 and L2 frequencies, see Table 1, have been considered. The undifferenced 

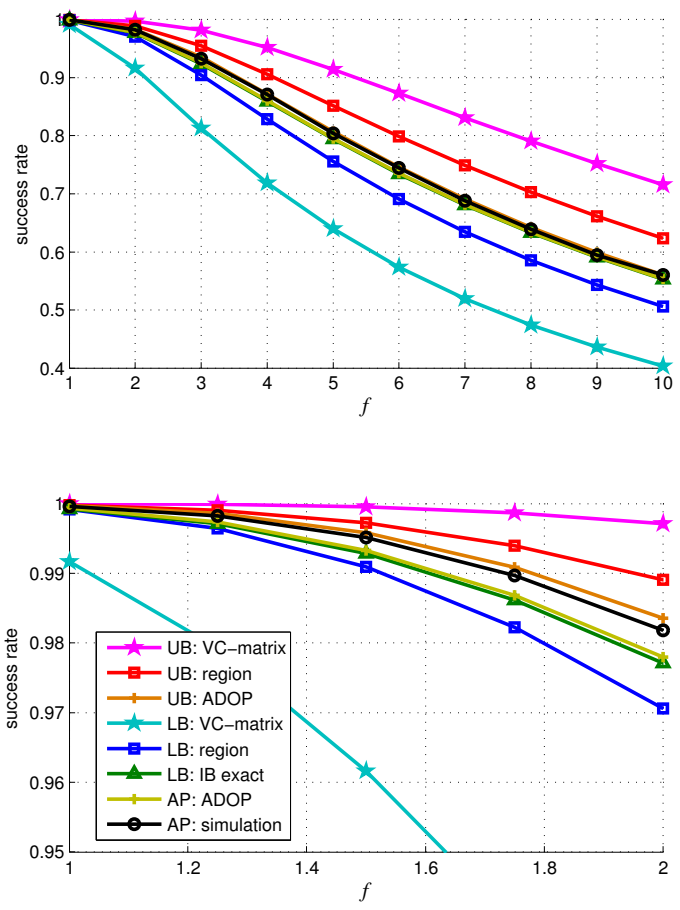

Figure 17: ILS success rate bounds for 2-frequency geometry-free model with 2 ambiguities, $f$ is the scale factor applied to the VC-matrix (bottom panel shows same results, but only for smaller $f)$. 
code and phase standard deviations were set to $15 \mathrm{~cm}$ and $1.5 \mathrm{~mm}$, respectively. The float ambiguity VC-matrix (units are $c y c l e s^{2}$ ) obtained in this way is:

$$
\boldsymbol{Q}_{\hat{a} \hat{a}}=\left[\begin{array}{ll}
1.2429 & 0.9683 \\
0.9683 & 0.7547
\end{array}\right]
$$

In addition, a scaling is applied to analyze the performance for different precisions:

$$
Q_{\hat{a} \hat{a}, f}=f \times Q_{\hat{a} \hat{a}}
$$

The ILS success rate approximations and bounds are shown in Figure 17 as a function of the scale factor $f$. The lower bound based on the exact IB success rate is very sharp. Interestingly, this also holds for the ADOP-based upper bound and approximation (the orange line is hardly visible, as it is plotted below the graph of the simulation-based success rate). In this case the bounds based on bounding the integration region are quite sharp if the success rate is high, but become less tight as the scale factor increases, and consequently the success rate decreases.

In all results shown so far, the bounds based on bounding the VC-matrix $\boldsymbol{Q}_{\hat{a} \hat{a}}$ are generally not tight at all. An example where also these bounds will work well is when all variances are equal to a certain value $v$ and all the covariances equal to a value $c$, with $v>>c$ :

$$
\sigma_{\hat{a}_{i} \hat{a}_{i}}^{2}=v, \sigma_{\hat{a}_{i} \hat{a}_{j}}=c, \quad \forall i, j=1, \ldots, n ; i \neq j
$$

Figure 18 shows the bounds for an example with $n=2, v=0.02$ and $c=0.0005$. Again the scaling according to Eq.(39) is applied.

\section{Which bounds or approximations to use?}

The results in this section show that the success rate bounds and approximations differ in their performance. The simulation-based approximations of the IR 


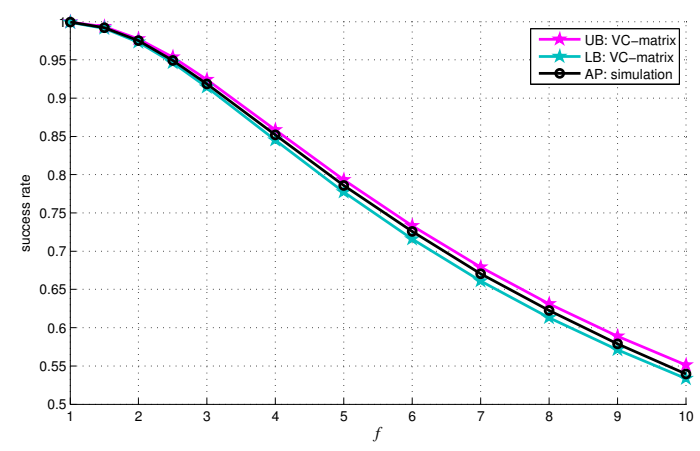

Figure 18: ILS success rate bounds based on bounding the $(2 \times 2)$ scaled VC-matrix with both variances equal to 0.02 , and covariance equal to 0.0005 . The scale factor is equal to $f$.

and ILS success rates work well if enough samples are used. However, they may not be suitable for real-time applications as their computation time may be long. Computation time will also be an issue for real-time applications if the upper bound of the ILS success rate based on bounding the integration is considered. For design and research purposes, as well as for post-processing, computation time will not be an issue. All other bounds and approximations can be used in real-time.

For the IR success rate, the lower bound was shown to perform well. For the ILS success rate, the lower bound based on the exact IB success rate, and the upper bound based on bounding the integration region generally perform very well for the GNSS models considered here. Furthermore, it was shown that the other bounds and approximations may work well for certain applications where the dimension is lower or the structure of the VC-matrix $\boldsymbol{Q}_{\hat{a} \hat{a}}$ is different, see for example Figures 17 and 18. 


\section{Success rates with GPS, Galileo and Compass}

As an example on how the Ps-LAMBDA toolbox can be used to assess the (potential) performance of GNSS, a comparative study will be presented for different GNSSs. Such a study is useful to:

- study the performance as obtained with the actual constellations for GPS and Compass;

- investigate what the potential of Galileo is, both as a stand-alone system or combined with GPS. The full nominal Galileo constellation (as planned) is used.

The current constellation of Galileo comprises only four satellites, and is therefore not considered in this study.

Here, the ILS success rates are evaluated for 25 different times on 22-March2012, 0:00 - 12:00 UTC, for a 35km baseline in Perth, Australia. The same assumptions for noise as in Table 3 are used for all signals. The standard deviation of the zenith ionosphere corrections is $15 \mathrm{~mm}$, zenith troposphere delays are estimated. The following signals, see Table 1, are considered:

- GPS L1+L5;

- Galileo L1+L5;

- Compass B1+B2;

- GPS+Galileo L1+L5/E5a. 

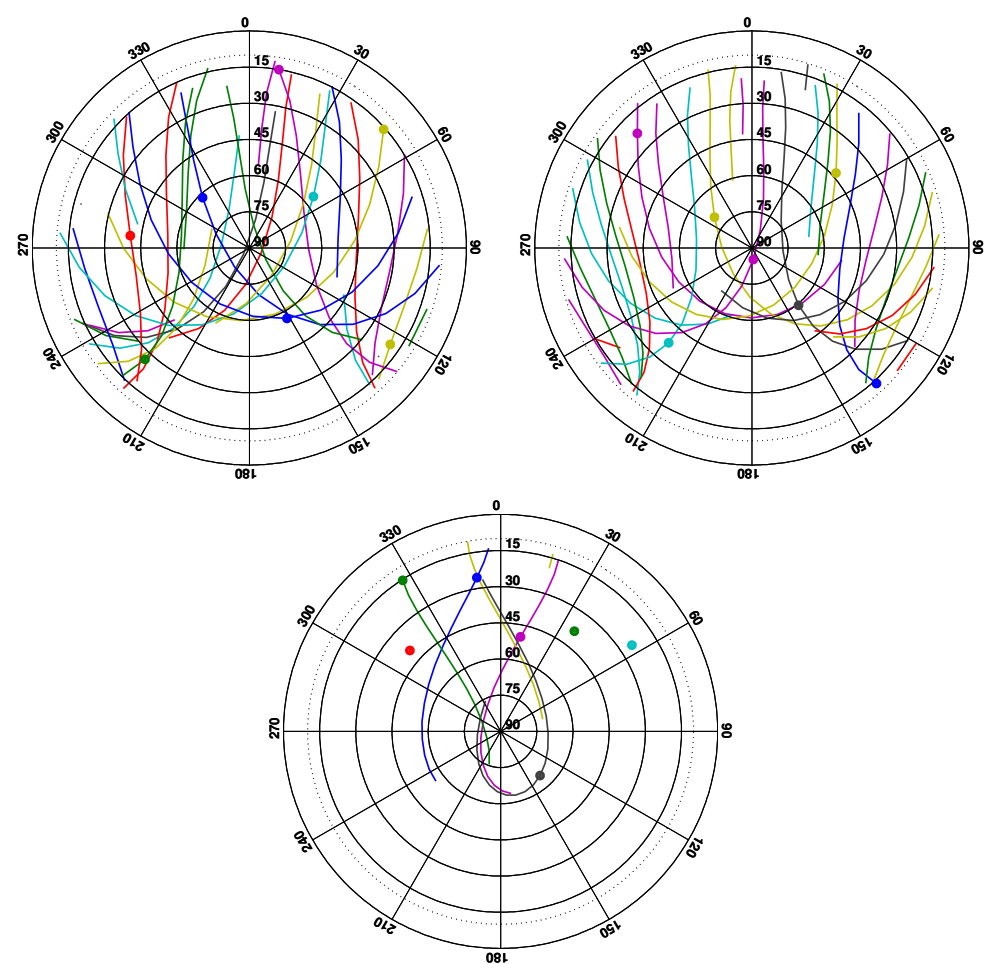

Figure 19: Skyplots of GPS (left), Galileo (middle) and Compass (right) for Perth, Australia on 22-March-2012, 0:00 - 12:00 UTC. The plots show the azimuth [deg] and elevation [deg] of the satellites; the dots correspond to the satellite positions at 6:00 UTC.

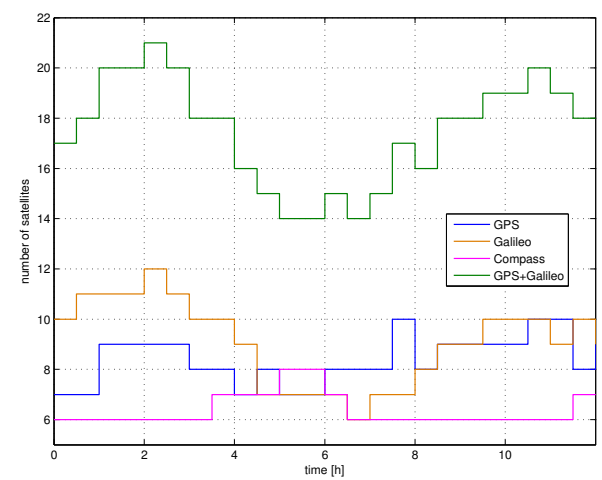

Figure 20: Number of visible satellites in Perth, Australia on 22-March-2012, 0:00 - 12:00 UTC. 
Using almanac data for each of the GNSSs, satellite positions can be computed and the matrices $\boldsymbol{A}$ and $\boldsymbol{B}$ in model (3) can be constructed, as well as the corresponding VC-matrix $\boldsymbol{Q}_{\boldsymbol{y} \boldsymbol{y}}$. Figure 19 shows the skyplots for the three considered constellations. Note that during this time window three geostationary satellites and five Inclined Geo-Synchronous Orbit satellites of Compass were visible. The GPS and Galileo constellations comprise of 32 and 30 Medium Earth Orbiting satellites at inclinations of 55 and 56 degrees, respectively. This explains the differences in Figure 19. Figure 20 shows the number of visible satellites as function of time for each system.

Figure 21 reports the following bounds and approximation of the ILS success rate: the lower bound based on IB, the upper bound based on bounding the integration region, and the simulation-based approximation with $10^{6}$ samples. The top and bottom panels show the single-epoch and four-epoch results, respectively. The single-epoch success rates are much lower (note the different scales in the bottom and top panels), and then the bounds are not as tight. However, when using 4 epochs of observations the success rates will improve significantly and also the bounds become much sharper.

These results can now be used to analyze and compare the performance of the different GNSSs.

\section{Satellite geometry and number of epochs}

In Section 4 an overview of the factors affecting the success rate was given. The results in Figure 21 clearly show some of these dependencies:

- Receiver-satellite geometry: success rates are generally higher if more satellites are visible, compare e.g. the number of visible Galileo satellites as 
function of time in Figure 20 with the 1-epoch success rates in Figure 21. However, even with the same number of visible satellites, the success rate may strongly fluctuate. See for example the results obtained for Compass: from 0:00 - 3:30 UTC six satellites are visible, but the 1-epoch success rate varies between 0.57 and 0.71 . This is due to the dependence of the success rate on the receiver-satellite geometry, since all other factors affecting the success rate remain the same.

- Number of observation epochs: more epochs will result in much improved success rates, as is clear by comparing the results from the top and bottom panels.

The dependency on measurement noise is not explicitly analyzed here, but the effect will be similar as when changing the number of observation epochs.

\section{Choice of GNSS}

For this scenario, GPS and Galileo would give similar performance. At times where more Galileo satellites are visible, the success rates with Galileo tend to be higher, as expected. Combined GPS+Galileo brings a great potential, as it significantly outperforms the single GNSSs.

The current Compass constellation provides on average 6 to 7 visible satellites at this location, which is generally lower than with the current GPS constellation. This causes the success rates to be lower on average. In addition, the receiversatellite geometry contributes to the lower success rates as well, since for Compass the satellite visibility is restricted to a smaller portion of the sky, as can be seen in Figure 19; with GPS and Galileo the satellites from Eastern and Western directions at different elevations will contribute to a better geometry. 

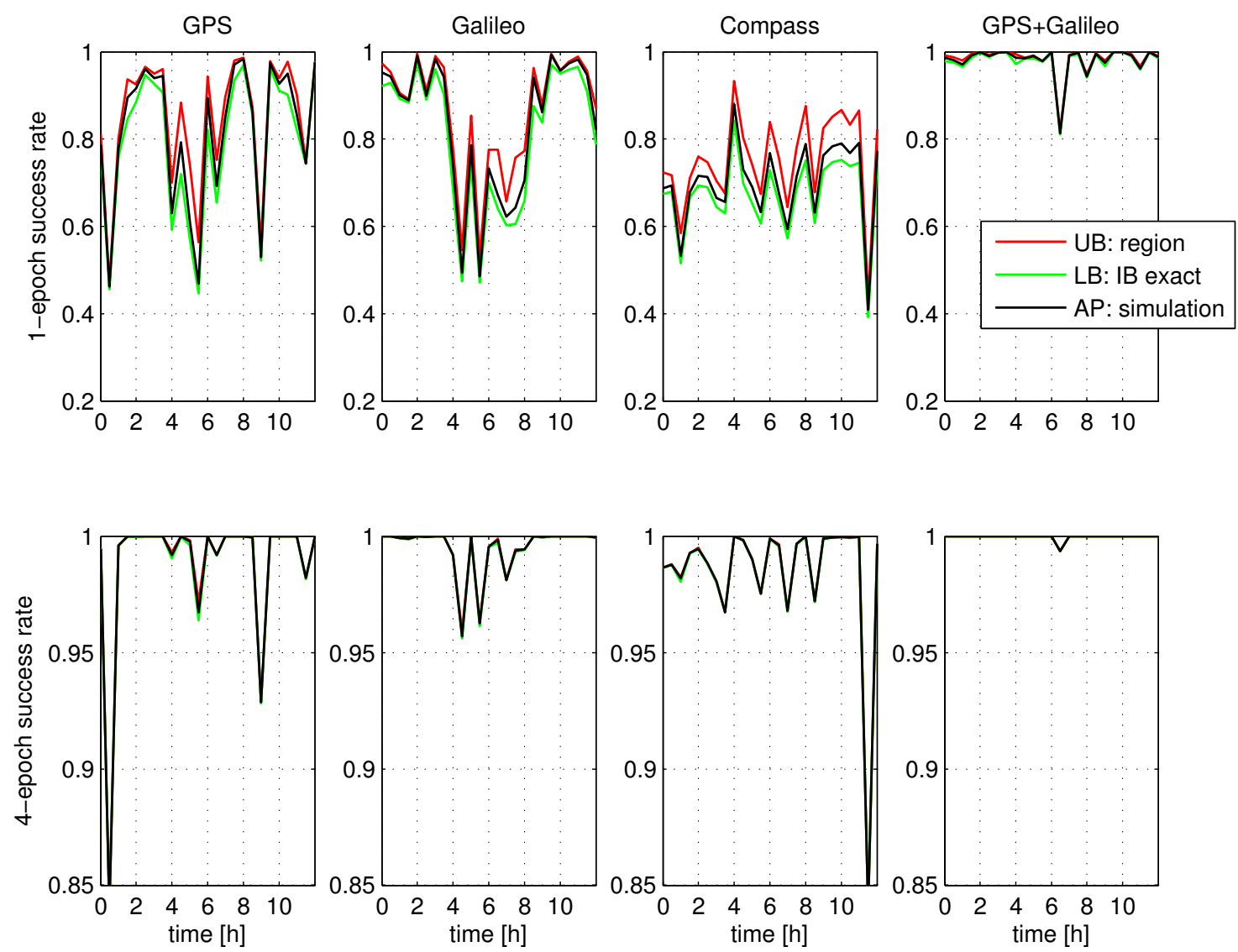

Figure 21: ILS success rates with GPS, Galileo, Compass or combined GPS+Galileo (from left to right) for a 35km baseline in Perth, Australia on 22-March-2012, 0:00 - 12:00 UTC. Top: 1-epoch model. Bottom: 4-epoch model.

\section{Further analysis}

The example shown here could easily be extended to study for example the benefit of having more Compass satellites available in the future, the effect of using different signals (i.e. frequencies), or the effect of different baseline lengths. 


\section{Concluding remarks}

In this contribution the Matlab toolbox Ps-LAMBDA is presented, which allows a user to compute different bounds and approximations of the success rate of integer estimation. All bounds and approximations from Section 5 have been included in the software since it was shown that it will depend very much on the model at hand which bounds and approximations are sharpest. By default the tool will calculate the exact IB success rate for the decorrelated ambiguities, since for GNSS models this provides a sharp lower bound to the ILS success rate, and an upper bound for the IR success rate.

We have focused here on GNSS models, but Ps-LAMBDA can be used for any integer estimation problem; the only input required is the variance-covariance matrix of the real-valued estimates of the integer parameters. As such, Ps-LAMBDA is a valuable tool for many applications that rely on the precise phase observations from GNSS or other interferometric techniques. Firstly, Ps-LAMBDA can be used for research and design purposes as to decide on which system and signals to use, to select the best time to take measurements, to know beforehand how many epochs of data will be required to ensure reliable ambiguity resolution, or to analyze whether successful ambiguity resolution for a given baseline length is feasible. Secondly, the tool can be used for deciding on acceptance of the integer ambiguity solution in real data processing.

In the presence of unmodeled biases, such as multipath, the probability of correct integer estimation will be negatively affected. For studying the biasrobustness or -sensitivity of ambiguity resolution, it will be useful to analyze the impact of a given bias on the success rate. A future version of the Ps-LAMBDA will therefore include an option to evaluate the bias-affected success rates as well. 
The Ps-LAMBDA toolbox is available upon request from the authors.

\section{Acknowledgments}

This work has been executed in the framework of the Positioning Program Project 1.01 New carrier phase processing strategies for achieving precise and reliable multi-satellite, multi-frequency GNSS/RNSS positioning in Australia of the Cooperative Research Centre for Spatial Information, whose activities are funded by the Australian Commonwealth's Cooperative Research Centres Programme.

Peter J.G. Teunissen is the recipient of an Australian Research Council (ARC) Federation Fellowship (project number FF0883188). All this support is gratefully acknowledged.

Bevis, M., Businger, S., Herring, T. A., Rocken, C., Anthes, R. A., Ware, R. H., 1992. GPS meteorology: remote sensing of atmospheric water vapor using the Global Positioning System. Journal of Geophysical Research 97, 15787-15801.

Blewitt, G., 1989. Carrier phase ambiguity resolution for the global positioning system applied to geodetic baselines up to $2000 \mathrm{~km}$. Journal of Geophysical Research 94, 135 - 151.

Bock, Y., 1996. Medium distance GPS measurements. In: PJG Teunissen and A Kleusberg, GPS for Geodesy, Springer-Verlag,Berlin.

Buist, P., Teunissen, P., Giorgi, G., Verhagen, S., 2010. Multivariate Bootstrapped Relative Positioning of Spacecraft Using GPS L1/Galileo E1 Signals. Advances in Space Research 47 (5), 770-785. 
Chang, X., Yang, X., Zhou, T., 2005. MLAMBDA: a modified LAMBDA method for integer least-squares estimation. Journal of Geodesy 79, 552-565.

Collins, P., Bisnath, S., Lahaye, F., Heroux, P., 2009. Undifferenced GPS ambiguity resolution using the decoupled clock model and ambiguity datum fixing. Navigation 57 (2), 123-135.

Dai, Z., Knedlik, S., Loffeld, O., 2009. Instantaneous triple-frequency GPS cycleslip detection and repair. International Journal of Navigation and Observation ID $407231,1-15$.

Das Neves Viegas, D. C., Cunha, S. R., 2007. Precise positioning by phase processing of sound waves. IEEE Trans. Signal Process. 55 (12), 5731-5738.

De Jonge, P. J., Tiberius, C., 1996. The LAMBDA method for integer ambiguity estimation: implementation aspects, LGR-Series, No 12. Tech. rep., Delft University of Technology.

deLacy, M. C., Reguzzoni, M., Sansò, F., 2011. Real-time cycle slip detection in triple-frequency GNSS. GPS Solutions Online first, 1-10.

Dong, D., Bock, Y., 1989. Global positioning system network analysis with phase ambiguity resolution applied to crustal deformation studies in California. Journal of Geophysical Reseach 94 (B4), 3949-3966.

Euler, H. J., Seeger, S., Zelzer, O., Takac, F., Zebhauser, B. E., 2004. Improvement of Positioning Performance Using Standardized Network RTK Messages. In: Proc. of ION NTM 2004, San Diego CA. pp. 453-461. 
Everaerts, J., 2008. The use of Unmanned Aerial Vehicles (UAVS) for remote sensing and mapping. The International Archives of the Photogrammetry, Remote Sensing and Spatial Information Sciences XXXVII (B1), 1187-1192.

Fernandes, R. M. S., Ambrosius, B. A. C., Noomen, R., Bastos, L., Miranda, L. C. M., Spakman, W., 2004. Angular velocities of Nubia and Somalia from continuous GPS data: implications on present-day relative kinematics. Earth and Planetary Science Letters 222, 197-208.

Geng, J., Shi, C., Ge, M., Dodson, A., Lou, Y., Zhao, Q., Liu, J., 2012. Improving the estimation of fractional-cycle biases for ambiguity resolution in precise point positioning. Journal of Geodesy 86, 579-589.

URL http://dx.doi.org/10.1007/s00190-011-0537-0

Hanssen, R. F., Teunissen, P. J. G., Joosten, P., 2001. Phase ambiguity resolution for stacked radar interferometric data. Proc. of the International Symposium on Kinematic Systems in Geodesy, GeomaticsandNavigation,Banff, Canada, 317320.

Hassibi, A., Boyd, S., 1998. Integer parameter estimation in linear models with applications to GPS. IEEE Transactions on Signal Processing 46 (11), 2938 -2952 .

Heroux, P., Kouba, J., 1995. GPS precise point positioning with a difference. In: Geomatics 95, Ottawa, Ontario, Canada.

Hobiger, T., Sekido, M., Koyama, Y., Kondo, T., 2009. Integer phase ambiguity estimation in next-generation geodetic Very Long Baseline Interferometry. Advances in Space Research 43, 187-192. 
Hofmann-Wellenhof, B., Lichtenegger, H., Collins, J., 2001. Global positioning system: theory and practice, 5th edn. Springer Berlin Heidelberg, New York.

Hofmann-Wellenhof, B., Lichtenegger, H., Wasle, E., 2008. GNSS global navigation systems: GPS, GLONASS, Galileo and More. Springer, Wien.

Huber, S., Younis, M., Krieger, G., 2010. The TanDEM-X mission: overview and interferometric performance. International Journal of Microwave and Wireless Technologies 2, 379-389.

Kondo, K., 2003. Optimal success/error rate and its calculation in resolution of integer ambiguities in carrier phase positioning of Global Positioning System (GPS) and Global Navigation Satellite System (GNSS). In: Proc. of ION Annual Meeting, Albuquerque NM. pp. 176-187.

Kroes, R., Montenbruck, O., Bertiger, W., Visser, P., 2005. Precise GRACE baseline determination using GPS. GPS Solutions 9 (1), 21-31.

Laurichesse, D., Mercier, F., Berthias, J. P., Broca, P., Cerri, L., 2009. Integer ambiguity resolution undifferenced GPS phase measurements and its application to PPP and satellite precise orbit determination. Navigation 56 (2), 135-149.

Leick, A., 2004. GPS satellite surveying. 3rd edn. John Wiley, New York.

Leung, S., Montenbruck, O., 2005. Real-Time Navigation of formation flying spacecraft using GPS measurements. Journal of Guidance, Control, and Dynamics 28 (2), 226-235.

Li, B., Teunissen, P. J. G., 2011. High dimensional integer ambiguity resolution: 
A first comparison between LAMBDA and Bernese. The Journal of Navigation 64, S192-S210.

Michel, G. W., Yu, Y. Q., Zhu, S. Y., Reigber, C., Becker, M., ReinhartandWSimons, E., Ambrosius, B., Vigny, C., Chamot-Rooke, N., Le PichonandPMorgan, X., Matheussen, S., 2001. Crustal motion and block behaviour in SE-Asia from GPS measurements. Earth and Planetary Science Letters 187, 239-244.

Misra, P., Enge, P., 2001. Global Positioning System: Signals, Measurements, and Performance. Ganga-Jamuna Press, Lincoln MA.

Nadarajah, N., Teunissen, P. J. G., Buist, P. J., 2012. Attitude determination of LEO satellites using an array of GNSS sensors. Accpeted for publication in Proc. of IEEE Fusion.

Odijk, D., 2002. Fast precise GPS positioning in the presence of ionospheric delays. Ph.D. thesis, Publications on Geodesy, 52, Netherlands Geodetic Commission,Delft.

Odijk, D., Teunissen, P. J. G., 2008. ADOP in closed form for a hierarchy of multifrequency single-baseline GNSS models. Journal of Geodesy 82, 473-492.

Odijk, D., Teunissen, P. J. G., Zhang, B., 2012. Single-frequency integer ambiguity resolution enabled precise point positioning. Journal of Surveying Engineering 2012, DOI:10.1061/(ASCE)SU.1943-5428.0000085.

Rieke, M., Foerster, T., Geipel, J., Prinz, T., 2011. High-precision positioning and real-time data processing of UAV-systems. The International Archives of the Photogrammetry, Remote Sensing and Spatial Information Sciences XXXVIII (1-C22), 1-6. 
Schon, S., Brunner, F. K., 2008. Atmospheric turbulence theory applied to GPS carrier-phase data. Journal of Geodesy 82, 47-57.

Strang, G., Borre, K., 1997. Linear Algebra, Geodesy, and GPS. WellesleyCambridge Press, Wellesley MA.

Takac, F., Zelzer, O., 2008. The Relationship Between Network RTK Solutions MAC, VRS, PRS, FKP and i-MAX. In: Proc. of ION GNSS 2008, Savannah GA. pp. 348-355.

Teunissen, P. J. G., 1993. Least squares estimation of the integer GPS ambiguities. In: Invited lecture, Section IV Theory and Methodology, IAG General Meeting, Beijing.

Teunissen, P. J. G., 1994. A new method for fast carrier phase ambiguity estimation. In: Proceedings IEEE Position, Location and Navigation Symposium PLANS'94. Las Vegas, NV, pp. 562-573.

Teunissen, P. J. G., 1995a. The invertible GPS ambiguity transformations. Manuscripta Geodaetia 20, 489-497.

Teunissen, P. J. G., 1995b. The least-squares ambiguity decorrelation adjustment: a method for fast GPS integer ambiguity estimation. Journal of Geodesy 70, $65-82$.

Teunissen, P. J. G., 1997. A canonical theory for short GPS baselines. Part IV: Precision versus reliability. Journal of Geodesy 71, 513-525.

Teunissen, P. J. G., 1998a. On the integer normal distribution of the GPS ambiguities. Artificial Satellites 33 (2), 49-64. 
Teunissen, P. J. G., 1998b. Success probability of integer GPS ambiguity rounding and bootstrapping. Journal of Geodesy 72, 606-612.

Teunissen, P. J. G., 1999a. An optimality property of the integer least-squares estimator. Journal of Geodesy 73 (11), 587-593.

Teunissen, P. J. G., 1999b. The probability distribution of the GPS baseline for a class of integer ambiguity estimators. Journal of Geodesy 73, $275-284$.

Teunissen, P. J. G., 2000. ADOP based upperbounds for the bootstrapped and the least-squares ambiguity success rates. Artificial Satellites 35 (4), 171-179.

Teunissen, P. J. G., Kleusberg, A., 1998. GPS for geodesy, 2nd edn. Springer Berlin Heidelberg New York.

Teunissen, P. J. G., Odijk, D., 1997. Ambiguity Dilution of Precision: definition, properties and application. In: Proc. of ION GPS-1997, Kansas City MO. pp. 891-899.

Thomsen, H. F., 2000. Evaluation of upper and lower bounds on the success probability. Proc. of ION GPS-2000, Salt Lake City UT, 183-188.

Todorova, S., Hobiger, T., Schuh, H., 2008. Using the Global Navigation Satellite System and satellite altimetry for combined Global Ionosphere Maps. Advances in Space Research, 727-736.

Van der Hoeven, A., Hanssen, R. F., Ambrosius, B., 2002. Tropospheric delay estimation and analysis using GPS and SAR interferometry. Physics and Chemistry of the Earth 27, 385-390. 
Verhagen, S., 2005. On the reliability of integer ambiguity resolution. Navigation $52(2), 99-110$.

Wickert, J., Schmidt, T., Michalak, G., Heise, S., Arras, C., Falck, G. B. C., König, R., Pingel, D., Rothacher, M., 2009. GPS Radio Occultation with CHAMP, GRACE-A, SAC-C, TerraSAR-X, and FORMOSAT-3/COSMIC: Brief Review of Results from GFZ. In: New Horizons in Occultation Research. A. K. Steiner, B. Pirscher, U. Foelsche, G. Kirchengast (Eds.). Springer-Verlag, Berlin, pp. 315.

Wu, Y., Jin, S. G., Wang, Z. M., Liu, J. B., 2010. Cycle-slip detection using multi-frequency GPS carrier phase observations: a simulation study. Advances in Space Research 46, 144-149.

Zumberge, J. F., Heflin, M. B., Jefferson, D. C., Watkins, M. M., Webb, F. H., 1997. Precise point positioning for the efficient and robust analysis of GPS data from large networks. Journal of Geophysical Research 102, 5005-5017. 


\section{List of Figures}

1 Position errors in East (dE), North $(\mathrm{dN})$ and $\mathrm{Up}(\mathrm{dU})$ direction in meters for ambiguity float solutions (top panel), ambiguity fixed solutions (bottom panel). Note the different scales in the top and bottom panels. . . . . 10

2 Scatterplot of horizontal position errors in meters for float solution (grey dots) and corresponding fixed solution. In this case, $93 \%$ of the solutions were correctly fixed (green dots), and 7\% was wrongly fixed (red dots). . 12

3 2D Pull-in regions for integer rounding (IR) and 50,000 float solutions. Top: original ambiguities $\hat{\boldsymbol{a}}$ [cycles]; Bottom: $\boldsymbol{Z}$-decorrelated ambiguities $\hat{z}$ [cycles]. . . . . . . . . . . . . . . . . . . . . 15

4 2D pull-in regions for integer bootstrapping (IB) and 50,000 float solutions. Top: original ambiguities $\hat{\boldsymbol{a}}$ [cycles]; Bottom: $\boldsymbol{Z}$-decorrelated ambiguities $\hat{z}$ [cycles] . . . . . . . . . . . . . . . . . . . . . . 17

5 2D pull-in regions for integer least squares (ILS) and 50,000 float solutions. Top: original ambiguities $\hat{\boldsymbol{a}}$ [cylces]; Bottom: $\boldsymbol{Z}$-decorrelated ambiguities $\hat{z}$ [cylces].

6 Top: PDF and 2D pull-in regions for ILS. Bottom: corresponding probability mass function. . . . . . . . . . . . . . . . . . 22

7 Ps-LAMBDA: overview of available methods and options in routine SuccessRate. Default option is indicated with $(*)$. Names of underlying routines are shown as well. $\mathrm{AP}=$ approximation (blue), $\mathrm{LB}=$ lower bound (green), $\mathrm{UB}=$ upper bound (red)

8 Examples of simulation-based success rate as function of number of samples. Each panel shows the results for a different GNSS model. . . . . . . 28

9 IR success rates: upper bound based on IB (red) and lower bound based on diagonal VC-matrix (green) versus the actual IR success rate for the models from Table $3 . \ldots \ldots \ldots$. . . . . . . . . . . . . . 31 
10 IB success rates: ADOP-based upper bound versus the exact IB success rate for the models from Table $3 \ldots \ldots \ldots$. . . . . . . . . . . 31

11 ILS success rates: lower bound based on IB (green) and upper bound based on ADOP (red) versus the actual ILS success rate for the models

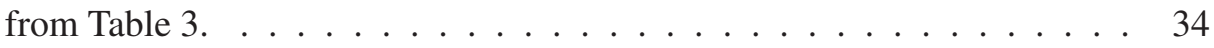

12 ILS success rates: ADOP-based approximation versus the actual ILS success rate for the models from Table $3 . \ldots \ldots \ldots$

13 Two examples of the ellipsoidal region (green) contained by the pull-in region $\mathcal{P}_{\mathbf{0} \text {,ILS }}$ (different shape of pull-in regions is due to different VCmatrices $\left.\boldsymbol{Q}_{\hat{z} \hat{z}}\right) \ldots \ldots \ldots \ldots \ldots$

14 Integration region (red) containing $\mathcal{P}_{\mathbf{0}, \text { ILS }}$ and defined by the intersection of two banded subsets. . . . . . . . . . . . . . . . . 36

15 ILS success rates: lower and upper bounds based on bounding the integration region versus the actual ILS success rate for the models from Table 3.

16 ILS success rates: lower and upper bounds based on bounding the VCmatrix versus the actual ILS success rate for the models from Table 3. . .

17 ILS success rate bounds for 2-frequency geometry-free model with 2 ambiguities, $f$ is the scale factor applied to the VC-matrix (bottom panel shows same results, but only for smaller $f$ ) . . . . . . . . . . . . 40

18 ILS success rate bounds based on bounding the $(2 \times 2)$ scaled VC-matrix with both variances equal to 0.02 , and covariance equal to 0.0005 . The scale factor is equal to $f \ldots \ldots \ldots \ldots \ldots \ldots$

19 Skyplots of GPS (left), Galileo (middle) and Compass (right) for Perth, Australia on 22-March-2012, 0:00 - 12:00 UTC. The plots show the azimuth [deg] and elevation [deg] of the satellites; the dots correspond to the satellite positions at 6:00 UTC. 
20 Number of visible satellites in Perth, Australia on 22-March-2012, 0:00 $12: 00$ UTC . . . . . . . . . . . . . . . . . . . . . . 44

21 ILS success rates with GPS, Galileo, Compass or combined GPS+Galileo (from left to right) for a $35 \mathrm{~km}$ baseline in Perth, Australia on 22-March2012, 0:00 - 12:00 UTC. Top: 1-epoch model. Bottom: 4-epoch model. . 47 


\section{List of Tables}

1 Overview of GNSS frequencies of open signals. . . . . . . . . . . 6

2 Percentage of float solutions that is correctly fixed for the three integer estimation methods (corresponding to Figures 3 to 5). . . . . . . . 20

3 Measurement scenarios used in Section 5 (standard deviations apply to zenith direction). . . . . . . . . . . . . . . . . . . 27 